\title{
On the equivalence between traction- and stress-based approaches for the modeling of localized failure in solids
}

\author{
Jian-Ying $\mathrm{Wu}^{\mathrm{a}, *}$, Miguel Cervera ${ }^{\mathrm{b}}$ \\ ${ }^{a}$ State Key Laboratory of Subtropic Building Science, South China Univeristy of Technology, 510641 Guangzhou, China. \\ ${ }^{b}$ CIMNE, Technical University of Catalonia, Edificio C1, Campus Norte, Jordi Girona 1-3, 08034 Barcelona, Spain.
}

\begin{abstract}
This work investigates systematically traction- and stress-based approaches for the modeling of strong and regularized discontinuities induced localized failure in solids. Two complementary methodologies, i.e., traction-based discontinuities localized in an elastic solid and strain localization of a stress-based inelastic softening solid, are addressed. In the former it is assumed a priori that the discontinuity forms with a continuous stress field and along the known orientation. A traction-based failure criterion is introduced to characterize the discontinuity and the orientation is determined from Mohr's maximization postulate. If the displacement jumps are retained as independent variables, the strong/regularized discontinuity approaches follow, requiring constitutive models for both the bulk and discontinuity. Elimination of the displacement jumps at the material point level results in the embedded/smeared discontinuity approaches in which an overall inelastic constitutive model fulfilling the static constraint suffices. The second methodology is then adopted to check whether the assumed strain localization can occur and identify its consequences on the resulting approaches. The kinematic constraint guaranteeing stress boundedness and continuity upon strain localization is established for general inelastic softening solids. Application to a unified elastoplastic damage model naturally yields all the ingredients of a localized model for the discontinuity (band), justifying the first methodology. Two dual but not necessarily equivalent approaches, i.e., the traction-based elastoplastic damage model and the stress-based projected discontinuity model, are identified. The former is equivalent to the embedded and smeared discontinuity approaches, whereas in the later the discontinuity orientation and associated failure criterion, not given $a$ priori, are determined consistently from the kinematic constraint. The bi-directional connections and equivalence conditions between the traction- and stress-based approaches are classified. Closed-form results under plane stress condition are also given. A generic failure criterion of either elliptic, parabolic or hyperbolic type, is analyzed in a unified manner, with the classical von Mises $\left(J_{2}\right)$, Drucker-Prager, Mohr-Coulomb and many other frequently employed criteria recovered as its particular cases.
\end{abstract}

Keywords:

Localized failure; strain localization; constitutive behavior; discontinuities; fracture; plasticity; damage.

\footnotetext{
* Tel.: (+86) 20-87112787

Email address: jywu@scut.edu.cn (Jian-Ying Wu)
} 


\section{Introduction}

Overall responses of inelastic softening solids are characterized by strain localization, i.e. a manifestation of concentration of micro-structural defects. Depending on the material of interest, the phenomena resulting from strain localization may be diverse: dislocations of order of microns in crystal metals, cracks of order of millimeters in concrete, and shear bands of order ranging from millimeters to kilometers in granular and geological problems. From the structural point of view these localization band may be regarded as a fracture surface of small or even negligible width compared to the length scale of the structure. Structural collapse is often induced by formation of such localization bands. Therefore, it is of utmost significance to evaluate (residual) structural safety once strain localization occurs, and to prevent potential catastrophic collapse caused by localized failure. However, despite the recent progresses made, the modeling of strain localization and subsequent structural collapse still remains a challenging issue.

Strain localization inevitably induces strain/displacement discontinuities, hindering the applicability of classical continuum mechanics. With respect to the strategies for the approximation of such discontinuities and resulting

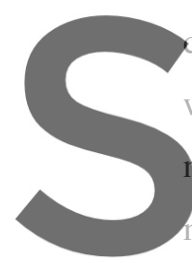

consequences on material

work of $\mathrm{Ngo}$ and Scordelis

onlinear fracture mechanic b
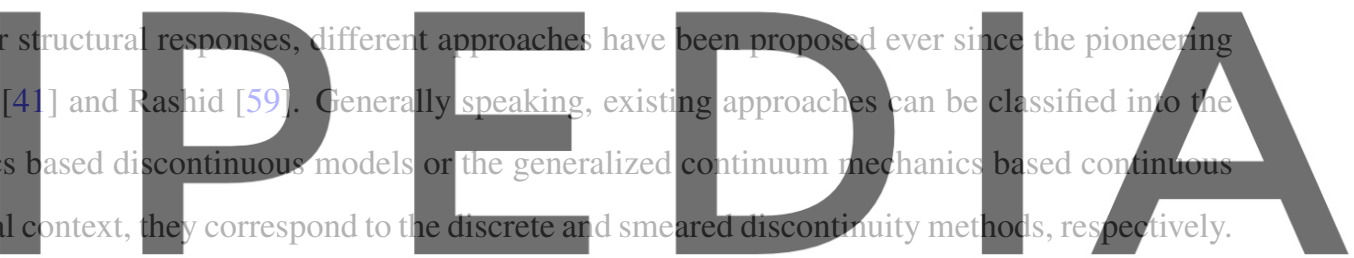

In the discontinuous (discrete) approach strain/displacement jumps are explicitly accounted for by embedding

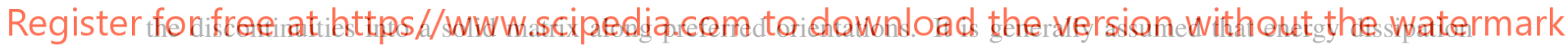

mechanisms are localized into the discontinuities while the bulk remains elastic. The traction continuity condition is imposed between them. The overall inelastic behavior is of anisotropy by construction. To characterize the dissipative behavior lumped in the discontinuities, vectorial traction-based cohesive models furnished with the fracture energy are introduced. Generally, displacement discontinuities are regarded as zero-width failure surfaces characterized by tractions vs. displacement jumps [3, 4, 22, 27]. Alternatively, strain discontinuities across the localized band with a finite width can be represented in terms of tractions vs. inelastic deformations (i.e., apparent displacement jumps normalized with respect to the bandwidth) $[13,14]$. Depending on the recoverable/irreversible properties of the discontinuities, traction-based cohesive models of either plastic [9, 70, 73], damage [1, 2, 29] or combined plasticdamage $[76,80]$ type can be established.

Contrariwise, the continuous approach relies on the introduction of a generalized continuum, so that stress-based constitutive models with regularized softening regime can be used. In this approach, the strain/displacement discontinuities are regularized (smeared) so that the classical concepts of (average) stress and strain still apply. It is no longer necessary to make distinction between the elastic bulk and the inelastic localization band. But rather, the overall nonlinear behavior of the weakened medium is described by generalized constitutive laws in terms of stress vs. strain 
tensors equipped with softening internal variables. In this way, plasticity and damage mechanics or their combination $[20,30]$ can be employed to develop appropriate inelastic constitutive laws. Induced anisotropy can be considered either theoretically $[24,36,80]$ or in the computational context $[16,17]$. Furthermore, to guarantee objectivity of the energy dissipated during the fracture process, the softening regime has to be regularized with respect to the length scale of localization band. The fracture energy - a material property measuring the dissipation per fracture surface, and an appropriately identified localization band width [5, 43], are fundamental ingredients for this purpose.

In the traction-based approached for the modeling of localized failure in solids, a crucial step is to determine the discontinuity orientation. This is a non-trivial goal for a new or propagating discontinuity whose orientation is not pre-defined or known a priori. To this end, ad hoc strategies have to be introduced, usually in an heuristic manner. For instance, the maximum tensile stress (i.e., Rankine) criterion is often adopted for mode I failure in quasi-brittle materials. For more general cases, selecting the discontinuity orientation according to some makeshift condition and fixing it afterwards becomes a superimposed condition on the material behavior. Fortunately, the occurrence of necessary requisites for a certain type of failure to be initiated during the whole deformation process in inelastic solids provides useful information. In this aspect, the pioneering works by Hill [25, 26], Thomas [72] and Rice [60, 65] have been widely adopted in the literature. The sufficient and necessary conditions for discontinuous bifurcation and strain

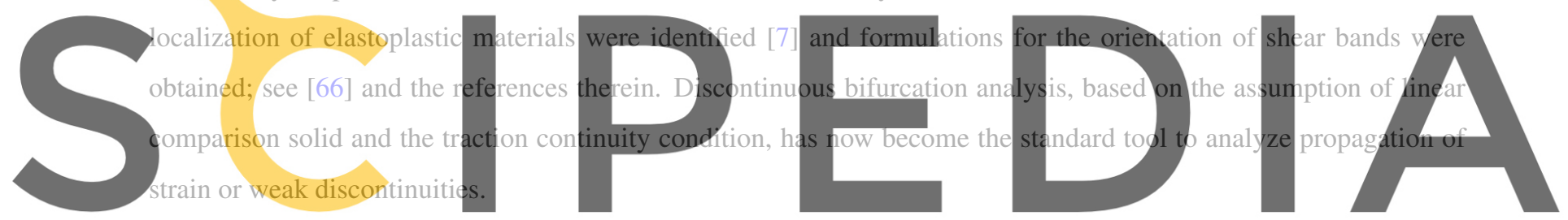

For strong discontinuities in solids with strain softening regimes, there is no consensus for the determination of the

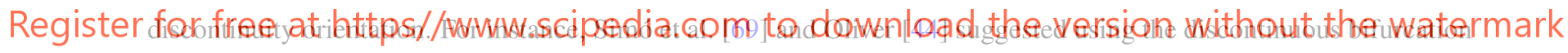

condition with perfect (null) softening/hardening modulus to determine the discontinuity orientation. However, this condition in general does not guarantee the occurrence of strong discontinuities [45, 46]. To remedy this problem, a variable band width model was proposed in the last reference, so that weak discontinuities can evolve smoothly to a strong one at a later stage. However, for strain localization to occur in a softening solid and develop eventually into a fully softened discontinuity at the final stage of the deformation process, material points inside the discontinuity (band) undergo inelastic loading while those outside it unload elastically. Therefore, provided the strong discontinuity cannot be guaranteed, the limit decohesion is not achieved in general cases and stress locking occurs due to the misprediction of the discontinuity orientation. Furthermore, due to the singular strain field associated with displacement discontinuities, traction continuity alone is not sufficient to guarantee physically meaningful results, but rather, stress boundedness also has to be invoked [44-47, 69]. Noticing this fact, Cervera et al. [15] recently used the kinematic compatibility condition resulting from stress boundedness to determine the discontinuity orientation, so that the stress locking-free property can be guaranteed for a fully softening discontinuity. The analytical results, obtained for von Mises $\left(J_{2}\right)$ and Drucker-Prager plastic materials in the cases of plane stress and plane strain, were validated by numerical simulations $[15,18]$. 
The failure criterion is another indispensable ingredient for the modeling of localized failure in solids. It defines an elastic domain outside which nonlinear energy dissipation mechanisms are active. In discontinuous approaches a traction-based failure criterion is in general adopted. For mode-I failure a trivial Rankine criterion can be employed. For mixed mode failure, the simple linear Mohr-Coulomb criterion is in general insufficient and more complex function has to be introduced, usually in an ad hoc manner. For instance, elliptical [8, 29, 57, 80], parabolic [39, 42] or hyperbolic $[9,19,40,73]$ functions have been postulated in the literature to describe mixed-mode failure in quasibrittle materials. However, the involved parameters are essentially mesoscopical entities hard to be determined from rather limited experimental tests. This shortcoming restrains heavily the application of traction-based discontinuous approaches. On the other hand, situations are totally different for the continuous approaches. Large quantities of stress-based failure criteria have been suggested based on triaxial test data, especially for concrete [20].

Traction-based discontinuity methods and stress-based material models with regularized softening regime both have been widely adopted for the modeling of localized failure in solids, though they are motivated from different methodologies and usually regarded as unconnected ones. It would be of great significance to establish a solid theoretical connection between results obtained from strain localization analysis of stress-based inelastic materials and from traction-based discontinuities localized into the elastic matrix. Such a correspondence will serve as a guidance

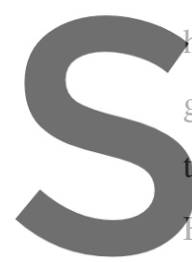

how to select an appropriate as

gap in identifying those mesor

ained macroscopic properties

For instance, Planas et al. [58]
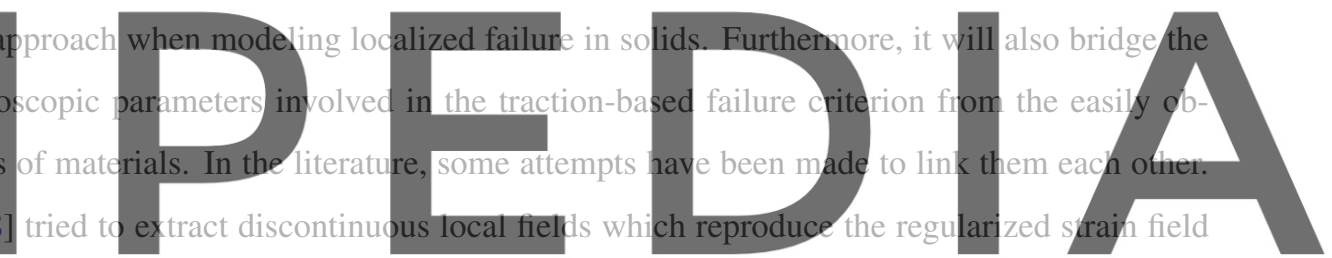

of a nonlocal continuum model; Mazars and Pijaudier-Cabot [35] suggested to seek a cohesive crack model which

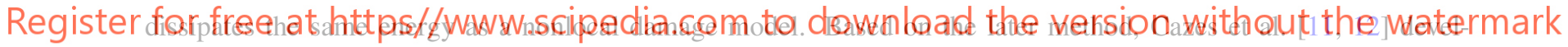

oped cohesive zone models which are energetically equivalent to non-local and gradient-enhanced continuum models.

Wells and Sluys [74] investigated the relation between the smeared crack model and the embeded one in a numerical context. Oliver and coworkers [45-49] derived the traction-based discontinuity models by projecting inelastic stressbased constitutive laws onto the discontinuity orientation. They managed deriving in closed-form the traction-based failure criterion for the isotropic damage model in total form [47-49]. Except for the Rankine and plane strain von Mises criteria [45, 46], stress-based plastic and plastic-damage models with general failure criterion have not been sufficiently accounted for. More importantly, the discontinuity orientation is determined through the discontinuous bifurcation condition. Accordingly, the resulting traction-based discontinuity model is in general inconsistent with the original stress-based counterpart and some mismatches are observed in the discontinuity kinematics [49, 50].

Recent investigations [77, 78] show that upon strain localization the stress field not only is bounded, but also is continuous, provided that relative rigid body motions (translations and rotations) are considered across the discontinuity [76]. Satisfaction of such a stress boundedness/continuity condition upon strain localization not only allows the formation of a fully softened discontinuity, but also establishes a bridge connecting the stress- and traction-based approaches motivated from rather different viewpoints. 
The objective of this work is to make further contributions to the above topics. Two complementary methodologies for the modeling of both strong and regularized discontinuities, i.e., traction-based discontinuities localized in an elastic solid and strain localization of a stress-based inelastic solid, are presented. Both formulations yield a fully softened discontinuity upon increasing deformations, with complete stress relaxation. This feature cannot be guaranteed with other procedures. Regarding the strategies dealing with the orientation and failure criterion of the discontinuity, the connections and in particular, the equivalence conditions, between traction- and stress-based approaches are investigated systematically. Note that the theoretical connections are bi-directional rather than uni-directional as in the existing literature. The established bi-directional connections between the traction and stress-based approaches make it possible to bridge the gap in-between them. Moreover, unified models that consider both elastic degradation and plastic deformations are addressed, with respect to a generic failure criterion which incorporates many classical ones as its particular cases. Particularly, a hyperbolic criterion appropriate for the modeling of localized failure in concrete is established, with the involved model parameters identified from available experimental data, so that the deficiency hindering its application in practice is largely facilitated.

The scope of the paper is restricted to the continuum setting. The interrelation with finite element approximations in the discrete setting is not covered in this work. Strong discontinuities, with discontinuous displacement fields,
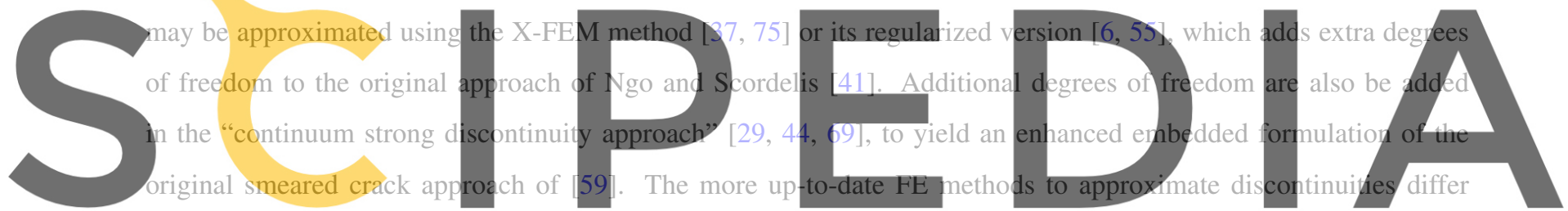

from the original ones in the enhanced discrete approximation of the (regularized or not) displacement jumps at the

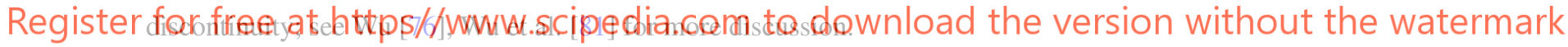

This paper is organized as follows. After this introduction, the kinematics and governing equations of localized failure in solids are briefly presented in Section 2. Within the first methodology of discontinuities localized in an elastic solid, several traction-based approaches are discussed in Section 3. To justify the first methodology, an alternative one, i.e., strain localization of a stress-based inelastic solid, is then considered in Section 4. The kinematic constraint guaranteeing the stress boundedness/continuity condition is derived for general inelastic softening solids and applied to a unified elastoplastic damage model. Two dual (formally identical) but not necessarily equivalent approaches, i.e., the traction-based elastoplastic damage model and the stress-based projected discontinuity model, are identified. Section 5 addresses the bi-directional connections, in particular, the equivalence conditions, between the aforementioned traction- and stress-based approaches. In Section 6 closed-form results in plane stress condition are given, with respect to a generic stress-based failure criterion. Some relevant conclusions drawn in Section 7 close the paper.

Notation. Compact tensor notation is used in this paper as far as possible. As a general rule, scalars are denoted by italic light-face Greek or Latin letters (e.g. $a$ or $\lambda$ ); vectors and second-order tensors are signified by italic boldface minuscule and majuscule letters like $\boldsymbol{a}$ and $\boldsymbol{A}$, respectively. Fourth-order tensors are identified by blackboard-bold majuscule characters (e.g. A). Symbols $I$ and II represent the second-order and symmetric fourth-order identity 
tensors, respectively. Superscripts 'T, and 'sym, indicate the transposition and symmetrization operations, respectively. The inner products with single and double contractions are denoted by ' $'$ ' and ' $?$ ', respectively. The dyadic product ' $\otimes$ ' and the symmetrized Kronecker product $\underline{\bar{\otimes}}$ are defined as

$$
(\boldsymbol{A} \otimes \boldsymbol{B})_{i j k l}=A_{i j} B_{k l}, \quad(\boldsymbol{A} \underline{\bar{\otimes}} \boldsymbol{B})_{i j k l}=\frac{1}{2}\left(A_{i k} B_{j l}+A_{i l} B_{j k}\right)
$$

\section{General formulation of localized failure in solids}

Let us consider the domain $\Omega \subset \mathbb{R}^{n_{\operatorname{dim}}}\left(n_{\operatorname{dim}}=1,2,3\right)$ shown in Fig. 1 . It is occupied by a solid with reference position vector $\boldsymbol{x} \in \mathbb{R}^{n_{\mathrm{dim}}}$. The boundary is denoted by $\Gamma \subset \mathbb{R}^{n_{\mathrm{dim}}-1}$, with an external unit normal vector $\boldsymbol{n}^{*}$. Deformations of the solid are characterized by the displacement field $u: \Omega \rightarrow \mathbb{R}^{n_{\mathrm{dim}}}$ and the infinitesimal strain field $\epsilon:=\nabla^{\mathrm{sym}} u$, with $\nabla(\cdot)$ being the spatial gradient operator. The solid is subjected to a distributed body force $b^{*}: \Omega \rightarrow \mathbb{R}^{n_{\text {dim }}}$ per unit volume. Surface tractions $t^{*}: \Gamma_{t} \rightarrow \mathbb{R}^{n_{\text {dim }}}$ and displacements $u^{*}: \Gamma_{u} \rightarrow \mathbb{R}^{n_{\text {dim }}}$ are imposed on the disjoint and complementary parts $\Gamma_{t} \subset \Gamma$ and $\Gamma_{u} \subset \Gamma$ of the boundary $\Gamma$, respectively.

At the early stage of the deformation process, standard compatibility relations of a continuum medium apply. That is, both the displacement and strain fields are continuous and regular (bounded). Upon satisfaction of a specific

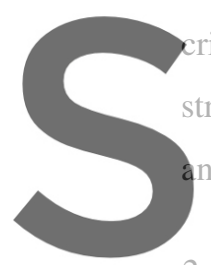
criterion, strain localization strong discontinuity or a reg is replaced by Maxwell

2.1. Kinematics of discontinuities
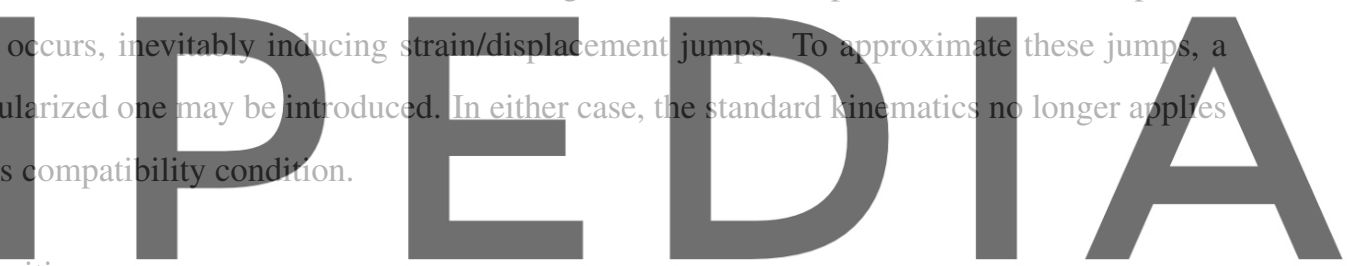

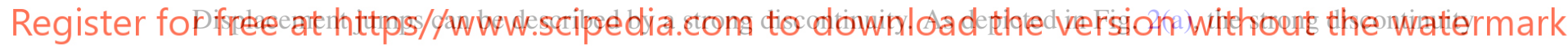

$\mathcal{S}$ splits the solid $\Omega$ into two parts $\Omega^{+}$and $\Omega^{-}$, located "ahead of" and "behind" $\mathcal{S}$, respectively, in such a way that $\Omega^{+} \cup \Omega^{-} \cup \mathcal{S}=\Omega$. The discontinuity orientation is denoted by a unit normal vector $\boldsymbol{n}$, pointing from $\Omega^{-}$to $\Omega^{+}$and fixed along time (i.e., $\dot{\boldsymbol{n}}=\boldsymbol{0})$. The strong discontinuity $\mathcal{S}$ causes displacement jumps $\boldsymbol{w}:=\boldsymbol{u}\left(\boldsymbol{x} \in \Omega^{+} \cap \mathcal{S}\right)-\boldsymbol{u}(\boldsymbol{x} \in$ $\left.\Omega^{-} \cap \mathcal{S}\right)$ across it. In this case, the displacement field $\boldsymbol{u}(\boldsymbol{x})$ can be expressed as

$\boldsymbol{u}(\boldsymbol{x})=\overline{\boldsymbol{u}}(\boldsymbol{x})+\mathscr{H}_{\mathcal{S}}(\boldsymbol{x}) \hat{\boldsymbol{u}}(\boldsymbol{x})$

so that the strain field $\boldsymbol{\epsilon}(\boldsymbol{x})$ is given by

$\boldsymbol{\epsilon}(\boldsymbol{x}):=\nabla^{\mathrm{sym}} \boldsymbol{u}(\boldsymbol{x})=\nabla^{\mathrm{sym}} \overline{\boldsymbol{u}}(\boldsymbol{x})+(\boldsymbol{w} \otimes \boldsymbol{n})^{\mathrm{sym}} \delta_{\mathcal{S}}(\boldsymbol{x})$

where $\overline{\boldsymbol{u}}(\boldsymbol{x})$ denotes the continuous part of the displacement field; $\mathscr{H}_{\mathcal{S}}(\boldsymbol{x})$ is Heaviside function defined at the interface $\mathcal{S}$, i.e., $\mathscr{H}_{\mathcal{S}}(\boldsymbol{x})=0$ if $\boldsymbol{x} \in \Omega^{-} \cup \mathcal{S}$ and $\mathscr{H}_{\mathcal{S}}(\boldsymbol{x})=1$ otherwise; $\delta_{\mathcal{S}}(\boldsymbol{x})$ denotes Dirac-delta at the discontinuity $\mathcal{S}$. By construction, the relative displacement field $\hat{\boldsymbol{u}}(\boldsymbol{x}): \Omega \rightarrow \mathbb{R}^{n_{\mathrm{dim}}}$, satisfying the property $\hat{\boldsymbol{u}}(\boldsymbol{x} \in \mathcal{S})=\boldsymbol{w}$, describes how the part $\Omega^{+}$moves as a relative rigid body (e.g. translations and rotations) with respect to the other one $\Omega^{-}$; see Wu [76]. Accordingly, though the relative displacement field $\hat{\boldsymbol{u}}(\boldsymbol{x})$ itself is not necessarily constant, its contribution 
to the strain field vanishes, i.e., $\nabla^{\mathrm{sym}} \hat{\boldsymbol{u}}(\boldsymbol{x})=\boldsymbol{0}$. If only relative rigid body translations are considered, the relative displacement field $\hat{\boldsymbol{u}}(\boldsymbol{x})$ is uniformly distributed and coincident with the displacement jump $\boldsymbol{w}$.

The unbounded strain field (2.1b) resulting from the discontinuous displacement field (2.1a) can be regularized over a discontinuity band $\mathcal{B}$ of finite width $b$. Here, the width $b$ is not a physical length but a regularization parameter which can be made as small as desired. As shown in Fig. 2(b), the regularized discontinuity (or discontinuity band) $\mathcal{B}$ is delimited by two surfaces $\mathcal{S}^{+}$and $\mathcal{S}^{-}$parallel to the discontinuity $\mathcal{S}$, i.e., $\Omega^{+} \cup \Omega^{-} \cup \mathcal{B}=\Omega$. In this case, the displacement field $\boldsymbol{u}(\boldsymbol{x})$ is continuous, with an apparent displacement jump $\boldsymbol{w}:=\boldsymbol{u}\left(\boldsymbol{x} \in \Omega^{+} \cap \mathcal{S}^{+}\right)-\boldsymbol{u}\left(\boldsymbol{x} \in \Omega^{-} \cap \mathcal{S}^{-}\right)$ across the discontinuity band $\mathcal{B}$. Accordingly, the $\mathcal{C}^{0}$-continuous displacement field $\boldsymbol{u}(\boldsymbol{x})$ can be expressed as

$\boldsymbol{u}(\boldsymbol{x})=\overline{\boldsymbol{u}}(\boldsymbol{x})+\mathscr{H}_{\mathcal{B}}(\boldsymbol{x}) \hat{\boldsymbol{u}}(\boldsymbol{x})$

and the singular strain field $(2.1 \mathrm{~b})$ is regularized as

$\boldsymbol{\epsilon}(\boldsymbol{x})=\nabla^{\mathrm{sym}} \overline{\boldsymbol{u}}(\boldsymbol{x})+(\boldsymbol{e} \otimes n)^{\mathrm{sym}} \Xi_{\mathcal{B}}(\boldsymbol{x})$

where the inelastic deformation vector $e:=w / b$ is defined as the apparent displacement jump $w$ normalized with respect to the band width $b ; \mathscr{H}_{\mathcal{B}}(x)$ is a regularized ramp function defined as $\mathscr{H}_{\mathcal{B}}(x)=0$ if $x \in \Omega^{-}, \mathscr{H}_{\mathcal{B}}(x)=$

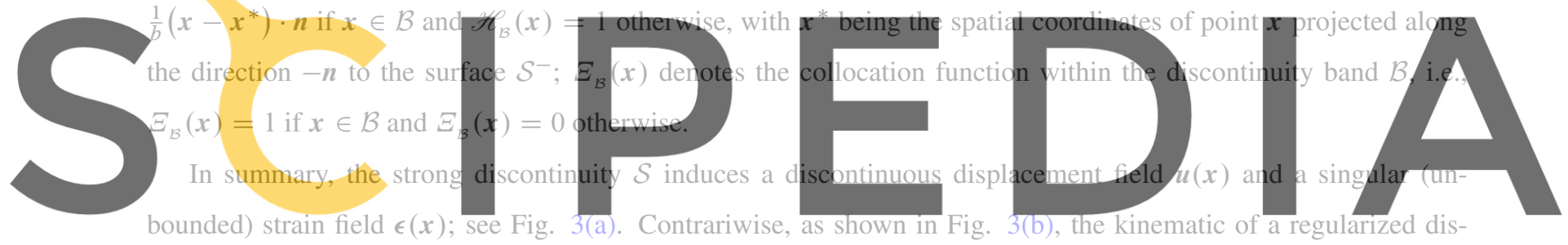

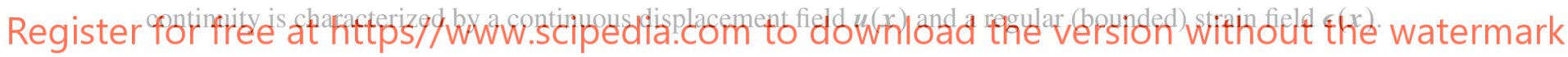

Remark 2.1. As the discontinuity band width $b$ tends to zero, it follows that

$\lim _{b \rightarrow 0} \mathscr{H}_{\mathcal{B}}(\boldsymbol{x})=\mathscr{H}_{\mathcal{S}}(\boldsymbol{x}), \quad \lim _{b \rightarrow 0} \frac{1}{b} \Xi_{\mathcal{B}}(\boldsymbol{x})=\delta_{\mathcal{S}}(\boldsymbol{x}), \quad \lim _{b \rightarrow 0} \boldsymbol{e} \Xi_{\mathcal{B}}(\boldsymbol{x})=\boldsymbol{w} \delta_{\mathcal{S}}(\boldsymbol{x})$

That is, the strong discontinuity can be regarded as the limit of a regularized one, with a vanishing band width $b \rightarrow 0$.

Reciprocally, a discontinuity band can be regarded as the convenient regularization of a strong discontinuity.

Remark 2.2 It is assumed in the kinematics (2.1) and (2.2) that the strains on both sides of the discontinuity are continuous, i.e., $\epsilon_{\mathcal{S}}^{+}=\epsilon_{\mathcal{S}}^{-}=\epsilon_{\mathcal{S}}^{ \pm}$, where $\epsilon_{\mathcal{S}}^{+}:=\boldsymbol{\epsilon}\left(\boldsymbol{x} \in \Omega^{+} \cap \mathcal{S}^{+}\right)$and $\epsilon_{\mathcal{S}}^{-}:=\boldsymbol{\epsilon}\left(\boldsymbol{x} \in \Omega^{-} \cap \mathcal{S}^{-}\right)$represent the strains "ahead of" the surface $\mathcal{S}^{+}$and "behind" the surface $\mathcal{S}^{-}$, respectively. Therefore, in this work no distinction is made between $\epsilon_{\mathcal{S}}^{+}$and $\epsilon_{\mathcal{S}}^{-}$, and are both denoted by $\epsilon_{\mathcal{S}}^{ \pm}$. The assumption disregards relative stretching that induces strain/stress differences across the discontinuity. Discontinuous bulk strains were consider in [51] and more recently, in [81], to find that such a relative stretching mode seldom dominates strain localization in softening solids. Contrariwise, the strain $\epsilon_{\mathcal{S}}:=\boldsymbol{\epsilon}(\boldsymbol{x} \in \mathcal{S})$ at the discontinuity (band) exhibits a jump with respect to the strain $\boldsymbol{\epsilon}_{\mathcal{S}}^{ \pm}$outside it, which verifies Maxwell's compatibility condition

$\llbracket \boldsymbol{\epsilon} \rrbracket:=\boldsymbol{\epsilon}_{\mathcal{S}}-\boldsymbol{\epsilon}_{\mathcal{S}}^{ \pm}=(\boldsymbol{e} \otimes \boldsymbol{n})^{\mathrm{sym}}=\frac{1}{b}(\boldsymbol{w} \otimes \boldsymbol{n})^{\mathrm{sym}}$ 
The strain jump $\llbracket \boldsymbol{\epsilon} \rrbracket$ is inversely proportional to $b$ for a regularized discontinuity (or unbounded for a strong one).

\subsection{Governing equations}

Restricting the discussion to quasi-static loading, the governing equations of the above problem are expressed as

Balance of linear momentum: $\quad \nabla \cdot \boldsymbol{\sigma}+\boldsymbol{b}^{*}=\boldsymbol{0} \quad$ in $\Omega \backslash \mathcal{S}$

Traction continuity condition: $\quad \boldsymbol{\sigma}_{\mathcal{S}}^{+} \cdot \boldsymbol{n}=\boldsymbol{\sigma}_{\mathcal{S}}^{-} \cdot \boldsymbol{n}=\boldsymbol{t} \quad$ in $\mathcal{S}$

subjected to the following boundary conditions

$\begin{array}{rll}\text { Traction boundary condition: } & \boldsymbol{\sigma} \cdot \boldsymbol{n}^{*}=\boldsymbol{t}^{*} & \text { in } \Gamma_{t} \\ \text { Displacement boundary condition: } & \boldsymbol{u}=\boldsymbol{u}^{*} & \text { in } \Gamma_{u}\end{array}$

where the second-order tensor $\sigma: \Omega \backslash \mathcal{S} \rightarrow \mathbb{R}^{n_{\mathrm{dim}} \times n_{\mathrm{dim}}}$ denotes the stress field in the bulk $\Omega \backslash \mathcal{S}=\Omega^{+} \cup \Omega^{-}$, with $\sigma_{\mathcal{S}}^{+}:=\sigma\left(x \in \Omega^{+} \cap \mathcal{S}\right)$ and $\sigma_{\mathcal{S}}^{-}:=\sigma\left(x \in \Omega^{-} \cap \mathcal{S}\right)$ being the stresses "ahead of" and "behind" the interface $\mathcal{S}$, respectively; the vector $t: \mathcal{S} \rightarrow \mathbb{R}^{n_{\mathrm{dim}}}$ represents the cohesive tractions transferred across the discontinuity $\mathcal{S}$.

3. Discontinuities localized in elastic solids

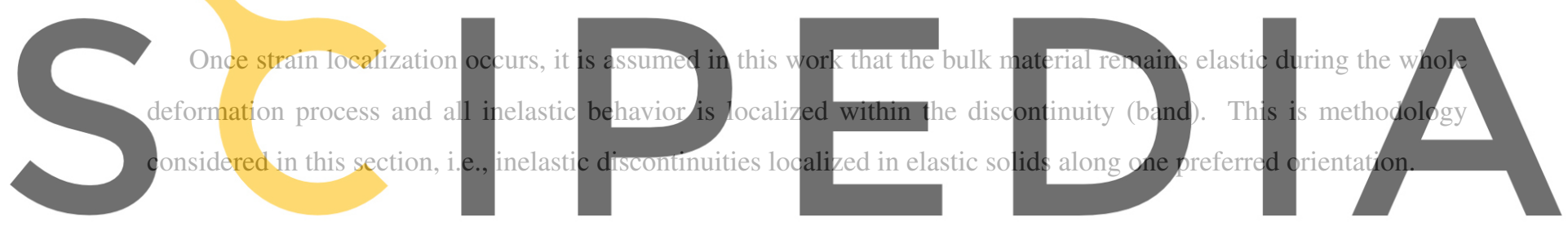

3.1. General framework

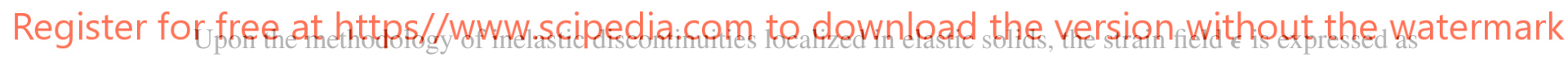

$\epsilon=\epsilon^{\mathrm{e}}+\epsilon^{\mathrm{in}}$

where $\epsilon^{\mathrm{e}}=\nabla^{\mathrm{sym}} \overline{\boldsymbol{u}}$ represents the elastic strain field; the inelastic strain field $\boldsymbol{\epsilon}^{\text {in }}$ is given by

$\boldsymbol{\epsilon}^{\text {in }}=(\boldsymbol{w} \otimes \boldsymbol{n})^{\mathrm{sym}} \delta_{\mathcal{S}} \quad$ or $\quad \boldsymbol{\epsilon}^{\text {in }}=(\boldsymbol{e} \otimes \boldsymbol{n})^{\mathrm{sym}} \boldsymbol{\Xi}_{\mathcal{B}}$

localized within the discontinuity (band).

Let us consider the following generic constitutive relations

$\sigma=\mathbb{E}^{0}: \epsilon^{\mathrm{e}}=\mathbb{E}^{0}:\left(\epsilon-\epsilon^{\mathrm{in}}\right), \quad \epsilon^{\mathrm{e}}=\epsilon-\epsilon^{\mathrm{in}}=\mathbb{C}^{0}: \sigma$

or equivalently, in rate form

$\dot{\boldsymbol{\sigma}}=\mathbb{E}^{0}: \dot{\boldsymbol{\epsilon}}^{\mathrm{e}}=\mathbb{E}^{0}:\left(\dot{\boldsymbol{\epsilon}}-\dot{\boldsymbol{\epsilon}}^{\mathrm{in}}\right), \quad \dot{\boldsymbol{\epsilon}}^{\mathrm{e}}=\dot{\boldsymbol{\epsilon}}-\dot{\boldsymbol{\epsilon}}^{\mathrm{in}}=\mathbb{C}^{0}: \dot{\boldsymbol{\sigma}}$

where $\mathbb{E}^{0}$ and $\mathbb{C}^{0}$ denote the fourth-order elastic stiffness and compliance tensors, respectively. Note that the constitutive relations (3.3) and (3.4) hold for both the elastic bulk and the discontinuity (band). 
Remark 3.1 As the inelastic strains $\epsilon^{\text {in }}$ are localized completely within the discontinuity (band), the elastic strain field $\boldsymbol{\epsilon}^{\mathrm{e}}$ is continuous in the entire solid. It follows from the constitutive relations (3.3) that, though the strain field may be discontinuous or even singular, the resulting stress filed is continuous across the discontinuity (band), i.e.,

$\sigma_{s}^{+}=\sigma_{s}^{-}=\sigma_{s}$

for the stress $\sigma_{\mathcal{S}}:=\sigma(x \in \mathcal{S})$ at the discontinuity (band). This conclusion, applicable for both strong and regularized discontinuities, is exactly the stress continuity condition upon strain localization in inelastic softening solids; see Wu and Cervera [79] for the details. In this case, the traction continuity condition $(2.5)_{2}$, which describes the relation between the bulk stresses $\sigma_{\mathcal{S}}^{+}=\sigma_{\mathcal{S}}^{-}$and tractions $\boldsymbol{t}$ transferred by the discontinuity (band), coincides with the classical static constraint at the material level, i.e.,

$t=\sigma_{S} \cdot n, \quad \dot{t}=\dot{\sigma}_{S} \cdot n$

where the material character $\dot{n}=0$ has been considered. The static constraint is employed in the embedded/smeared crack models $[2,63]$ and traction-based elastoplastic damage models [80] discussed later in this work.

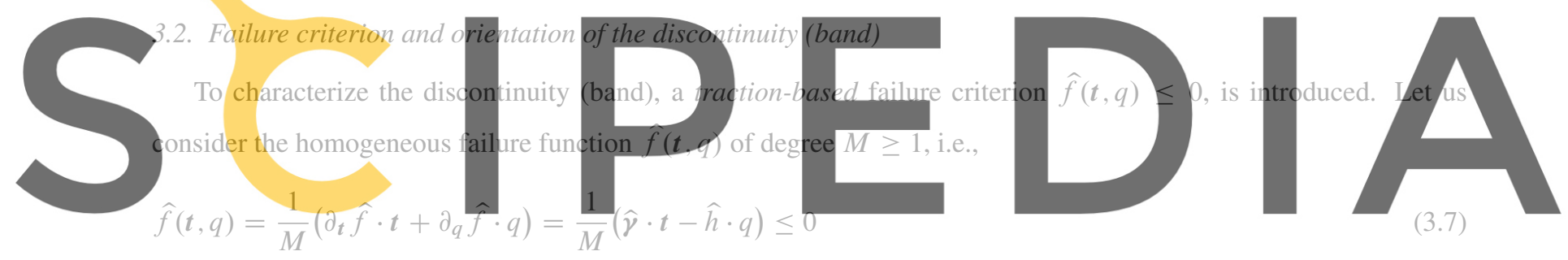

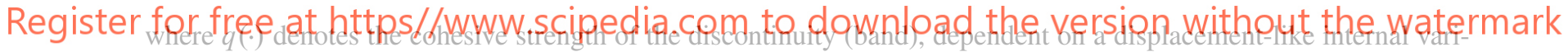

able $\tilde{\kappa}$ (or a strain-like one $\kappa$ ); $\hat{\gamma}:=\partial \hat{f} / \partial t$ and $\hat{h}:=-\partial \hat{f} / \partial q$ are the derivatives. As will be clear, the vector $\hat{\gamma}$

characterizes the evolution laws of the inelastic discontinuity (band).

The above failure criterion $\hat{f}(\boldsymbol{t}, q) \leq 0$ is expressed in terms of the traction $\boldsymbol{t}=\boldsymbol{\sigma} \cdot \boldsymbol{n}$ (hereafter the subscript ' $\mathcal{S}$ ' associated with $\boldsymbol{\sigma}_{\mathcal{S}}$ is dropped for brevity), or equivalently, of the stress tensor $\boldsymbol{\sigma}$ and the normal vector $\boldsymbol{n}$ of the discontinuity (band). Accordingly, the discontinuity orientation $\boldsymbol{n}$ has to be determined, usually in an heuristic manner (e.g., along the major principal stress for mode-I fracture) which might be insufficient for general cases.

Owing to the static constraint (3.6), Mohr's maximization postulate [38] is adopted in this work for a given traction-based failure criterion $\hat{f}(t, q) \leq 0$. That is, a discontinuity is initiated on the orientation upon which the tractions $\boldsymbol{t}\left(\boldsymbol{\theta}^{\mathrm{cr}}\right)$ maximize the failure function $\hat{f}[\boldsymbol{t}(\boldsymbol{\theta}), q]$, i.e.,

$\widehat{\boldsymbol{\theta}}^{\mathrm{cr}}=\arg \max \hat{f}[\boldsymbol{t}(\boldsymbol{\theta}), q] \quad \forall \boldsymbol{\theta}$

where the characteristic angles $\widehat{\boldsymbol{\theta}}^{\text {cr }}$ define the discontinuity orientation; $\boldsymbol{t}(\boldsymbol{\theta})$ denotes the traction vector acting on the surface with the normal $\boldsymbol{n}(\boldsymbol{\theta})$. 
Accordingly, the following stationarity condition holds

$\left.\frac{\partial \hat{f}}{\partial \boldsymbol{\theta}}\right|_{\widehat{\boldsymbol{\theta}}^{\mathrm{cr}}}=\left(\hat{\boldsymbol{\gamma}} \cdot \frac{\partial \boldsymbol{t}}{\partial \boldsymbol{\theta}}\right)_{\widehat{\boldsymbol{\theta}}^{\mathrm{cr}}}=\boldsymbol{O}$

together with a negative definite Hessian matrix

$\left.\boldsymbol{\theta}^{*} \cdot \frac{\partial^{2} \hat{f}}{\partial \boldsymbol{\theta}^{2}}\right|_{\hat{\boldsymbol{\theta}}^{\mathrm{cr}}} \cdot \boldsymbol{\theta}^{*}<0 \quad \forall \boldsymbol{\theta}^{*}$

The stationarity condition (3.9) results in a set of nonlinear equations so that the discontinuity angles $\hat{\boldsymbol{\theta}}^{\mathrm{cr}}$ can be solved.

\subsection{Strong/regularized discontinuity approaches}

With respect to the strategies dealing with the kinematic unknowns $w$ (or $e:=w / b$ ), two approaches can be identified from the methodology of inelastic discontinuities localized in elastic solids. Either the singular kinematics (2.1) or the regularized one (2.2) can be incorporated in both approaches.

Let us first consider the strong/regularized discontinuity approaches in which the kinematic unknowns $\boldsymbol{w}$ (or $\boldsymbol{e}$ ) associated to the discontinuity (band) are retained as independent variables. With the singular kinematics (2.1), the governing equations (2.5) and the traction boundary condition (2.6) 1 can be
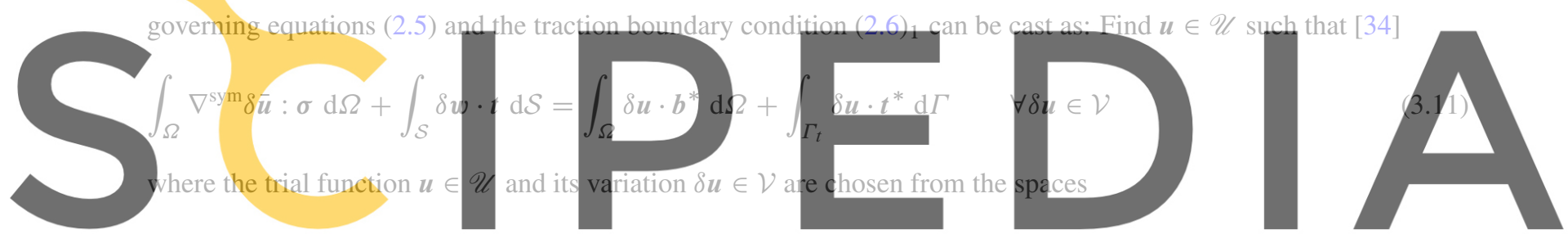

$\mathscr{U}:=\left\{u \mid u=\bar{u}+\mathscr{H}_{\mathcal{S}} \hat{u} ; \boldsymbol{u}=u^{*}\right.$ in $\Gamma_{u} ; \hat{u}=w$ in $\left.\mathcal{S}\right\}$

(3.12a)

Register for free at https//,www scipedia, com to download the version without the watermark

Alternatively, if the regularized kinematics (2.2) is employed, the weak form (3.11) becomes: Find $\boldsymbol{u} \in \mathscr{U}$ such that

$$
\int_{\Omega} \nabla^{\mathrm{sym}} \delta \overline{\boldsymbol{u}}: \boldsymbol{\sigma} \mathrm{d} \Omega+\int_{\mathcal{B}} \delta \boldsymbol{e} \cdot \boldsymbol{t} \mathrm{d} \mathcal{B}=\int_{\Omega} \delta \boldsymbol{u} \cdot \boldsymbol{b}^{*} \mathrm{~d} \Omega+\int_{\Gamma_{t}} \delta \boldsymbol{u} \cdot \boldsymbol{t}^{*} \mathrm{~d} \Gamma \quad \forall \delta \boldsymbol{u} \in \mathcal{V}
$$

where the trial function $\boldsymbol{u} \in \mathscr{U}$ and its variation $\delta \boldsymbol{u} \in \mathcal{V}$ are chosen within the spaces

$$
\begin{aligned}
\mathscr{U} & :=\left\{\boldsymbol{u} \mid \boldsymbol{u}=\overline{\boldsymbol{u}}+\mathscr{H}_{\mathcal{B}} \hat{\boldsymbol{u}} ; \boldsymbol{u}=\boldsymbol{u}^{*} \text { in } \Gamma_{u} ; \hat{\boldsymbol{u}}=\boldsymbol{w} \text { in } \mathcal{S}\right\} \\
\mathcal{V} & :=\left\{\delta \boldsymbol{u} \mid \delta \boldsymbol{u}=\delta \overline{\boldsymbol{u}}+\mathscr{H}_{\mathcal{B}} \delta \hat{\boldsymbol{u}} ; \delta \boldsymbol{u}=\boldsymbol{0} \text { in } \Gamma_{u} ; \delta \hat{\boldsymbol{u}}=\delta \boldsymbol{w} \text { in } \mathcal{S}\right\}
\end{aligned}
$$

In the weak form (3.11) or (3.13), the displacement boundary condition (2.6) 2 are enforced in strong form, and the variations $(\delta \overline{\boldsymbol{u}}, \delta \hat{\boldsymbol{u}})$ are of sufficient regularity.

\subsubsection{Localized plastic-damage models for the discontinuity (band)}

To proceed, the above weak form (3.11) or (3.13) has to be supplemented with constitutive relations for both the bulk stress $\sigma$ and tractions $\boldsymbol{t}$ transferred across the discontinuity (band). The linear elastic relation (3.3) is assumed for 
the bulk. As the tractions $\boldsymbol{t}$ are work conjugated to the displacement jumps $\boldsymbol{w}$ or the deformation vector $\boldsymbol{e}$, a cohesive model in terms of tractions versus displacement jumps (or inelastic deformations) is required.

Within the framework of irreversible thermodynamics, thermodynamically consistent cohesive models for the strong/regularized discontinuities can be developed; see Wu and Cervera [79] for the details. Here, only the necessary formulas are summarized in Table 1 and all the derivations are omitted.

Table 1: Cohesive models for the discontinuity (band)

\begin{tabular}{|c|c|c|c|}
\hline \multicolumn{2}{|l|}{ Cohesive model for the strong discontinuity } & \multicolumn{2}{|c|}{ Cohesive model for the regularized discontinuity } \\
\hline Kinematic decomposition & & Kinematic decomposition & \\
\hline $\begin{array}{l}\boldsymbol{w}=\boldsymbol{w}^{\mathrm{d}}+\boldsymbol{w}^{\mathrm{p}}, \quad \boldsymbol{\epsilon}^{\mathrm{in}}=\boldsymbol{\epsilon}^{\mathrm{d}}+\boldsymbol{\epsilon}^{\mathrm{p}} \\
\text { Constitutive relations }\end{array}$ & $(3.15)$ & $\begin{array}{l}\boldsymbol{e}=\boldsymbol{e}^{\mathrm{d}}+\boldsymbol{e}^{\mathrm{p}}, \quad \boldsymbol{\epsilon}^{\mathrm{in}}=\boldsymbol{\epsilon}^{\mathrm{d}}+\boldsymbol{\epsilon}^{\mathrm{p}} \\
\text { Constitutive relations }\end{array}$ & $(3.16)$ \\
\hline $\boldsymbol{t}=\widetilde{\boldsymbol{E}}^{\mathrm{d}} \cdot \boldsymbol{w}^{\mathrm{d}}=\widetilde{\boldsymbol{E}}^{\mathrm{d}} \cdot\left(\boldsymbol{w}-\boldsymbol{w}^{\mathrm{p}}\right)$ & (3.17a) & $\boldsymbol{t}=\boldsymbol{E}^{\mathrm{d}} \cdot \boldsymbol{e}^{\mathrm{d}}=\boldsymbol{E}^{\mathrm{d}} \cdot\left(\boldsymbol{e}-\boldsymbol{e}^{\mathrm{p}}\right)$ & (3.18a) \\
\hline $\boldsymbol{w}^{\mathrm{d}}=\boldsymbol{w}-\boldsymbol{w}^{\mathrm{p}}=\widetilde{\boldsymbol{C}}^{\mathrm{d}} \cdot \boldsymbol{t}$ & $(3.17 b)$ & $\boldsymbol{e}^{\mathrm{d}}=\boldsymbol{e}-\boldsymbol{e}^{\mathrm{p}}=\boldsymbol{C}^{\mathrm{d}} \cdot \boldsymbol{t}$ & $(3.18 b)$ \\
\hline $\begin{array}{l}\text { Rate form } \\
\dot{\boldsymbol{t}}=\widetilde{\boldsymbol{E}}^{\mathrm{d}} \cdot\left(\dot{\boldsymbol{w}}-\dot{\boldsymbol{w}}^{\mathrm{dis}}\right), \quad \dot{\boldsymbol{w}}=\widetilde{\boldsymbol{C}}^{\mathrm{d}} \cdot \dot{\boldsymbol{t}}+\dot{\boldsymbol{w}}^{\mathrm{dis}} \\
\text { Evolution laws }\end{array}$ & $(3.19)$ & $\begin{array}{l}\text { Rate form } \\
\dot{\boldsymbol{t}}=\boldsymbol{E}^{\mathrm{d}} \cdot\left(\dot{\boldsymbol{e}}-\dot{\boldsymbol{e}}^{\mathrm{dis}}\right), \quad \dot{\boldsymbol{e}}=\boldsymbol{C}^{\mathrm{d}} \cdot \dot{\boldsymbol{t}}+\dot{\boldsymbol{e}}^{\mathrm{dis}} \\
\text { Evolution laws }\end{array}$ & $(3.20)$ \\
\hline$\dot{\boldsymbol{w}}^{\mathrm{dis}}=\dot{\tilde{\boldsymbol{C}}}^{\mathrm{d}} \cdot \boldsymbol{t}+\dot{\boldsymbol{w}}^{\mathrm{p}}=\tilde{\lambda} \boldsymbol{\gamma}, \quad \dot{\tilde{\kappa}}=\tilde{\lambda} h$ & (3.21a) & $\dot{\boldsymbol{e}}^{\mathrm{dis}}=\dot{\boldsymbol{C}}^{\mathrm{d}} \cdot \boldsymbol{t}+\dot{\boldsymbol{e}}^{\mathrm{p}}=\lambda \boldsymbol{\gamma}, \quad \dot{\kappa}=\lambda h$ & $(3.22 \mathrm{a})$ \\
\hline$\dot{\boldsymbol{w}}^{\mathrm{p}}=(1-\xi) \dot{\boldsymbol{w}}^{\mathrm{dis}}=(1-\xi) \tilde{\lambda} \boldsymbol{\gamma}$ & $(3.21 b)$ & $\dot{\boldsymbol{e}}^{\mathrm{p}}=(1-\xi) \dot{\boldsymbol{e}}^{\mathrm{dis}}=(1-\xi) \lambda \boldsymbol{\gamma}$ & $(3.22 b)$ \\
\hline $\begin{array}{l}\dot{\tilde{\boldsymbol{C}}}^{\mathrm{d}}=\xi \tilde{\lambda} \frac{\boldsymbol{\gamma} \otimes \boldsymbol{\gamma}}{\boldsymbol{\gamma} \cdot \boldsymbol{t}} \\
\text { Kuhn-Tucker loading/unloading rules }\end{array}$ & $(3.21 \mathrm{c})$ & $\begin{array}{l}\dot{\boldsymbol{C}}^{\mathrm{d}}=\xi \lambda \frac{\boldsymbol{\gamma} \otimes \boldsymbol{\gamma}}{\boldsymbol{\gamma} \cdot \boldsymbol{t}} \\
\text { Kuhn-Tucker loading/unloading rules }\end{array}$ & $(3.22 \mathrm{c})$ \\
\hline $\begin{array}{l}\tilde{\lambda} \geq 0, \quad \hat{f} \leq 0, \quad \tilde{\lambda} \hat{f}=0 \\
\text { Consistency parameter }\end{array}$ & $(3.23)$ & $\begin{array}{l}\lambda \geq 0, \quad \hat{f} \leq 0, \quad \lambda \hat{f}=0 \\
\text { Consistency parameter }\end{array}$ & $(3.24)$ \\
\hline $\begin{array}{l}\tilde{\lambda}=\frac{\boldsymbol{\gamma} \cdot \widetilde{\boldsymbol{E}}^{\mathrm{d}} \cdot \dot{\boldsymbol{w}}}{\boldsymbol{\gamma} \cdot \widetilde{\boldsymbol{E}}^{\mathrm{d}} \cdot \boldsymbol{\gamma}+h \cdot \tilde{H} \cdot h}=\frac{\boldsymbol{\gamma} \cdot \dot{\boldsymbol{t}}}{h \cdot \tilde{H} \cdot h} \\
\text { Rate constitutive relations }\end{array}$ & $(3.25)$ & $\begin{array}{l}\lambda=\frac{\boldsymbol{\gamma} \cdot \boldsymbol{E}^{\mathrm{d}} \cdot \dot{\boldsymbol{e}}}{\boldsymbol{\gamma} \cdot \boldsymbol{E}^{\mathrm{d}} \cdot \boldsymbol{\gamma}+h \cdot H \cdot h}=\frac{\boldsymbol{\gamma} \cdot \dot{\boldsymbol{t}}}{h \cdot H \cdot h} \\
\text { Rate constitutive relations }\end{array}$ & $(3.26)$ \\
\hline $\begin{array}{l}\dot{\boldsymbol{t}}=\widetilde{\boldsymbol{E}}_{\mathrm{tan}}^{\mathrm{d}} \cdot \dot{\boldsymbol{w}}, \quad \dot{\boldsymbol{w}}=\widetilde{\boldsymbol{C}}_{\tan }^{\mathrm{d}} \cdot \dot{\boldsymbol{t}} \\
\text { Discontinuity tangents }\end{array}$ & $(3.27)$ & $\begin{array}{l}\dot{\boldsymbol{t}}=\boldsymbol{E}_{\mathrm{tan}}^{\mathrm{d}} \cdot \dot{\boldsymbol{e}}, \quad \dot{\boldsymbol{e}}=\boldsymbol{C}_{\tan }^{\mathrm{d}} \cdot \dot{\boldsymbol{t}} \\
\text { Discontinuity tangents }\end{array}$ & (3.28a) \\
\hline$\widetilde{\boldsymbol{E}}_{\mathrm{tan}}^{\mathrm{d}}=\widetilde{\boldsymbol{E}}^{\mathrm{d}}-\frac{\widetilde{\boldsymbol{E}}^{\mathrm{d}} \cdot(\boldsymbol{\gamma} \otimes \boldsymbol{\gamma}) \cdot \widetilde{\boldsymbol{E}}^{\mathrm{d}}}{\boldsymbol{\gamma} \cdot \widetilde{\boldsymbol{E}}^{\mathrm{d}} \cdot \boldsymbol{\gamma}+h \cdot \widetilde{H} \cdot h}$ & (3.29a) & $\boldsymbol{E}_{\mathrm{tan}}^{\mathrm{d}}=\boldsymbol{E}^{\mathrm{d}}-\frac{\boldsymbol{E}^{\mathrm{d}} \cdot(\boldsymbol{\gamma} \otimes \boldsymbol{\gamma}) \cdot \boldsymbol{E}^{\mathrm{d}}}{\boldsymbol{\gamma} \cdot \boldsymbol{E}^{\mathrm{d}} \cdot \boldsymbol{\gamma}+h \cdot H \cdot h}$ & (3.30a) \\
\hline$\widetilde{\boldsymbol{C}}_{\tan }^{\mathrm{d}}=\widetilde{\boldsymbol{C}}^{\mathrm{d}}+\frac{\boldsymbol{\gamma} \otimes \boldsymbol{\gamma}}{h \cdot \widetilde{H} \cdot h}$ & $(3.29 b)$ & $\boldsymbol{C}_{\tan }^{\mathrm{d}}=\boldsymbol{C}^{\mathrm{d}}+\frac{\boldsymbol{\gamma} \otimes \boldsymbol{\gamma}}{h \cdot H \cdot h}$ & $(3.30 \mathrm{~b})$ \\
\hline
\end{tabular}

In the kinematic decompositions (3.15) and (3.16), the displacement jump $\boldsymbol{w}$ (or the inelastic deformation vector $\boldsymbol{e}$ ) is split into a recoverable damage part $\boldsymbol{w}^{\mathrm{d}}$ (or $\boldsymbol{e}^{\mathrm{d}}$ ) and an unrecoverable plastic one $\boldsymbol{w}^{\mathrm{p}}$ (or $\boldsymbol{e}^{\mathrm{p}}$ ). Accordingly, both stiffness degradation and irreversible deformations induced by the discontinuity (band) can be accounted for in the constitutive relations (3.17) and (3.18), where the second-order tensors $\widetilde{\boldsymbol{E}}^{\mathrm{d}}$ (or $\boldsymbol{E}^{\mathrm{d}}$ ) and $\widetilde{\boldsymbol{C}}^{\mathrm{d}}$ (or $\boldsymbol{C}^{\mathrm{d}}$ ) denote the 
variable stiffness and compliance tensors of the discontinuity (band), respectively. In the rate forms (3.19) and (3.20), $\dot{\boldsymbol{w}}^{\mathrm{dis}}:=\dot{\widetilde{\boldsymbol{C}}}^{\mathrm{d}} \cdot \boldsymbol{t}+\dot{\boldsymbol{w}}^{\mathrm{p}}$ and $\dot{\boldsymbol{e}}^{\mathrm{dis}}:=\dot{\boldsymbol{C}}^{\mathrm{d}} \cdot \boldsymbol{t}+\dot{\boldsymbol{e}}^{\mathrm{p}}$ represent the dissipative displacement jump rate and dissipative deformation rate, respectively, both meaningful only in rate sense; see Figs. 4(a) and 4(b). The associated evolution laws (3.21) and (3.22) are both characterized by the dissipative flow vector $\gamma=\hat{\gamma}$ and softening function $h=\hat{h}$ which are the derivatives of the failure criterion (3.7).

In order to discriminate between damage and plastic contributions, a material parameter $\xi \in[0,1]$ is introduced. Namely, the cases $\xi=0$ and $\xi=1$ correspond to cohesive models of pure plastic and pure damage types, respectively. For the intermediate value $\xi \in(0,1)$, combined plastic-damage cohesive models follow. When the discontinuity (band) is inactive, i.e., $\hat{f}(\boldsymbol{t}, q)<0$, it follows from the Kuhr-Tucker loading/unloading rules (3.23) or (3.24) that $\tilde{\lambda}=0$ or $\lambda=0$; when the discontinuity (band) evolves, it follows that $\hat{f}(t, q)=0$. In the loading case, the Lagrangian multiplier $\tilde{\lambda}>0$ or $\lambda>0$ is solved from the consistency condition $\dot{\hat{f}}=0$ as that given in Eq. (3.25) or (3.26), where the softening moduli are given by $\widetilde{H}:=\partial q / \partial \tilde{\kappa}<0$ and $H:=\partial q / \partial \kappa<0$, with $\tilde{\kappa}$ and $\kappa$ being the displacement-like and strain-like internal variables, respectively. Finally, owing to the associated evolution laws, the discontinuity tangents (3.29) and (3.30) are all symmetric.

\subsubsection{Fracture energy and the equivalence conditions}

For a strong discontinuity, the fracture energy $G_{\mathrm{f}}$, usually regarded as a material property and defined as energy dissipation $W$ per unit of discontinuity area $A_{\mathcal{S}}$, can be evaluated as [79]

$G_{\mathrm{f}}:=\frac{W}{A_{\mathcal{S}}}=\left(1-\frac{1}{2} \xi\right) \int_{0}^{\infty} \tilde{\lambda} \gamma \mathrm{d} T=\left(1-\frac{1}{2} \xi\right) \int_{0}^{\infty} q(\tilde{\kappa}) \mathrm{d} \tilde{\kappa}$

where the evolution laws (3.21) and the relation $\hat{\gamma} \cdot \boldsymbol{t}=\hat{h} \cdot q$ for an active discontinuity (band) has been considered. Once the displacement-driven softening law $q(\tilde{\kappa})$ is given, the fracture energy $G_{\mathrm{f}}$ can be evaluated explicitly from Eq. (3.31). That is, the parameters involved in the softening law $q(\tilde{\kappa})$ can be determined for the given fracture energy $G_{\mathrm{f}}$.

Similarly, for the regularized discontinuity it follows that

$G_{\mathrm{f}}:=\frac{W}{A_{\mathcal{S}}}=\frac{W}{V_{\mathcal{B}} / b}=b\left(1-\frac{1}{2} \xi\right) \int_{0}^{\infty} \lambda \gamma \mathrm{d} T=b\left(1-\frac{1}{2} \xi\right) \int_{0}^{\infty} q(\kappa) \mathrm{d} \kappa$

for the volume $V_{\mathcal{B}}=b A_{\mathcal{S}}$ of the discontinuity band. It can be concluded that for the given fracture energy $G_{\mathrm{f}}$, the softening function $q(\kappa)$ also depends on the band width $b$.

The fracture energy (3.32) allows introducing the so-called specific fracture energy $g_{f}$, i.e., the dissipation per unit volume of the discontinuity band $V_{\mathcal{B}}$, in a straightforward manner

$g_{\mathrm{f}}:=\frac{W}{V_{\mathcal{B}}}=\int_{0}^{\infty} \sigma: \dot{\boldsymbol{\epsilon}} \mathrm{d} T=\frac{1}{b} G_{\mathrm{f}}$

This is exactly the relation between the crack band theory [5] and the fictitious crack model [27].

For the case of a vanishing band width $b \rightarrow 0$, the relation $g_{\mathrm{f}}=G_{\mathrm{f}} \delta_{\mathcal{S}}$ holds. It then follows that

$W=\int_{\mathcal{B}} g_{\mathrm{f}} \mathrm{d} \mathcal{B}=\int_{\mathcal{B}} G_{\mathrm{f}} \delta_{\mathcal{S}} \mathrm{d} \mathcal{B}=\int_{\mathcal{S}} G_{\mathrm{f}} \mathrm{d} \mathcal{S}$ 
which recovers the classical definition of fracture energy.

For the same traction-based failure criterion $\hat{f}(t, q) \leq 0$ and equivalent softening laws $q(\kappa)=q(\tilde{\kappa})$, the results (3.31) and (3.32) imply that identical fracture energies $G_{\mathrm{f}}$ can be obtained for both models, provided that

$\kappa=\frac{1}{b} \tilde{\kappa}, \quad \dot{\kappa}=\frac{1}{b} \dot{\tilde{\kappa}} \quad \Longleftrightarrow \quad \lambda=\frac{1}{b} \tilde{\lambda}, \quad \frac{1}{H}=\frac{1}{b} \frac{1}{\widetilde{H}}$

That is, the strong discontinuity transforms into an equivalent regularized counterpart with a finite band width $b \nrightarrow 0$.

Contrariwise, for the regularized discontinuity with a vanishing band width $b \rightarrow 0$, the conditions (3.35) become

$$
\kappa=\tilde{\kappa} \delta_{\mathcal{S}}, \quad \dot{\kappa}=\dot{\tilde{\kappa}} \delta_{\mathcal{S}} \quad \Longleftrightarrow \quad \lambda=\tilde{\lambda} \delta_{\mathcal{S}}, \quad \frac{1}{H}=\frac{1}{\widetilde{H}} \delta_{\mathcal{S}}
$$

In this case, the regularized discontinuity localizes into an equivalent strong discontinuity.

The above results, together with kinematic equivalence stated in Remark 2.1, clearly show that the strong and regularized discontinuity approaches are consistently related.

\subsection{Embedded/smeared discontinuity approaches}

Providing the cohesive model for the discontinuity (band) is known, it is possible to establish alternative embed$\mathrm{ded} /$ smeared discontinuity approaches. In such approaches, the weak form of the governing equation $(2.5)_{1}$ and the traction boundary condition (2.6) 1 can be re-stated as: Find $\boldsymbol{u} \in \mathscr{U}$ such that

$\int_{\Omega} \nabla^{\mathrm{sym}} \delta \boldsymbol{u}: \boldsymbol{\sigma} \mathrm{d} \Omega=\int_{\Omega} \delta \boldsymbol{u} \cdot \boldsymbol{b}^{*} \mathrm{~d} \Omega+\int_{\Gamma_{t}} \delta \boldsymbol{u} \cdot \boldsymbol{t}^{*} \mathrm{~d} \Gamma \quad \forall \delta \boldsymbol{u} \in \mathcal{V}$

with the stress $\sigma_{\mathcal{S}}$ at the discontinuity (band) determined from an inelastic material model satisfying the static constraint (3.6). Here, $\mathscr{U}$ is the trial space introduced in Eq. (3.12a) for a strong discontinuity or in Eq. (3.14a) for a regularized one; the standard test space $\mathcal{V}$ is defined as

$\mathcal{V}:=\left\{\delta \boldsymbol{u}\right.$ of sufficient regularity $\mid \delta \boldsymbol{u}=\boldsymbol{0}$ on $\left.\Gamma_{u}\right\}$

As can be seen, the traction continuity condition $(2.5)_{2}$ and the displacement jump $\boldsymbol{w}$ (or the inelastic deformation vector $\boldsymbol{e}$ ) are not explicitly considered in the weak form (3.37). But rather, they are accounted for indirectly at the local (material constitutive) level through the inelastic material models fulfilling the classical static constraint (3.6). Similarly, owing to the correspondence stated in Remark 2.1 and the equivalence demonstrated in Section 3.3.2, only the smeared discontinuity model employing the regularized kinematics (2.2) is addressed.

To derive the smeared discontinuity model, let us recall the constitutive relations (3.4) and (3.30b). Calling for the static constraint (3.6) $)_{2}$, the inelastic deformation vector rate $\dot{\boldsymbol{e}}$ is determined in terms of the strain rate $\dot{\boldsymbol{\epsilon}}$ as

$\dot{\boldsymbol{e}}=\left(\boldsymbol{E}_{\mathrm{tan}}^{\mathrm{d}}+\boldsymbol{n} \cdot \mathbb{E}^{0} \cdot \boldsymbol{n}\right)^{-1} \cdot\left(\mathbb{E}^{0}: \dot{\boldsymbol{\epsilon}}\right) \cdot \boldsymbol{n}$

Therefore, the stress rate $\dot{\boldsymbol{\sigma}}$ is given from Eq. (3.4), i.e.,

$\dot{\boldsymbol{\sigma}}=\mathbb{E}^{0}:\left[\dot{\boldsymbol{\epsilon}}-(\dot{\boldsymbol{e}} \otimes \boldsymbol{n})^{\mathrm{sym}}\right]=\mathbb{E}_{\mathrm{tan}}: \dot{\boldsymbol{\epsilon}}$ 
where the tangent stiffness tensor $\mathbb{E}_{\tan }$ is expressed as

$\mathbb{E}_{\tan }=\mathbb{E}^{0}-\mathbb{E}^{0}:\left[\left(\boldsymbol{E}_{\tan }^{\mathrm{d}}+\boldsymbol{n} \cdot \mathbb{E}^{0} \cdot \boldsymbol{n}\right)^{-1} \underline{\bar{\otimes}} \boldsymbol{N}\right]^{\mathrm{sym}}: \mathbb{E}^{0}$

with a second-order geometric tensor $N:=\boldsymbol{n} \otimes \boldsymbol{n}$.

Alternatively, it follows from the relation (3.28a) and the static constraint $(3.6)_{2}$ that

$\dot{\boldsymbol{\epsilon}}^{\mathrm{in}}=(\dot{\boldsymbol{e}} \otimes \boldsymbol{n})^{\mathrm{sym}}=\left[\left(\boldsymbol{C}_{\mathrm{tan}}^{\mathrm{d}} \cdot \dot{\boldsymbol{\sigma}} \cdot \boldsymbol{n}\right) \otimes \boldsymbol{n}\right]^{\mathrm{sym}}=\left(\boldsymbol{C}_{\tan }^{\mathrm{d}} \underline{\bar{\otimes}} \boldsymbol{N}\right)^{\mathrm{sym}}: \dot{\boldsymbol{\sigma}}$

The strain rate $\dot{\boldsymbol{\epsilon}}$ is given from Eq. (3.1)

$\dot{\boldsymbol{\epsilon}}=\dot{\boldsymbol{\epsilon}}^{\mathrm{e}}+\dot{\boldsymbol{\epsilon}}^{\mathrm{in}}=\mathbb{C}_{\mathrm{tan}}: \dot{\boldsymbol{\sigma}}$

for the tangent compliance $\mathbb{C}_{\tan }$ expressed as

$\mathbb{C}_{\tan }=\mathbb{C}^{0}+\left(\boldsymbol{C}_{\tan }^{\mathrm{d}} \underline{\bar{\otimes}} \boldsymbol{N}\right)^{\mathrm{sym}}$

Note that the tangent compliance $\mathbb{C}_{\text {tan }}$ can also be directly derived from Eq. (3.41) through the Sherman-MorrisonWoodburg formula [23].

The above constitutive relations correspond exactly to those in the smeared crack model [62-64], as an extension of the original form proposed by Rashid [59]. As can be seen, owning to stress continuity (3.5) upon strain localization, the kinematic unknowns $\boldsymbol{w}$ (or $\boldsymbol{e}:=\boldsymbol{w} / b$ ) can be eliminated at the local material-point level through the static constraint (3.6), resulting in a single constitutive model to describe the overall inelastic behavior of the solid.

Remark 3.2 Calling for the decomposition (3.15) or (3.16), the strain $\epsilon$ at the discontinuity (band) is additively split into its elastic, damage and plastic parts

$\epsilon=\epsilon^{\mathrm{e}}+\epsilon^{\mathrm{in}}=\epsilon^{\mathrm{e}}+\epsilon^{\mathrm{d}}+\epsilon^{\mathrm{p}}$

This is the kinematic decomposition suggested by Armero and Oller [2]. Accordingly, the above embedded/smeared discontinuity models fit the framework developed in that reference. As shown in next section, an equivalent tractionbased elastoplastic damage model can be developed based on an alternative kinematic decomposition.

\section{Strain localization of inelastic solids}

In the methodology employed in Section 3, it is implicitly assumed a priori that strain localization occurs with a continuous stress field and the discontinuity (band) orientation is known. However, on the one hand, whether such strain localization can occur has not been checked yet; even though it can, the effects of stress continuity on the discontinuity orientation have not been identified, either. On the other hand, the traction-based failure criterion characterizing the discontinuity (band) is usually introduced in a heuristic or even ad hoc manner, and it is hard to determine the involved parameters from available experimental data. 
In this section, an alternative methodology, i.e., strain localization in inelastic softening solids, is considered. As is well-known, for strain localization to occur in a softening solid and to develop eventually into a fully softened discontinuity at the final stage of the deformation process, material points inside the discontinuity (band) undergo inelastic loading while those outside it unload elastically [15, 45, 46]. This fact imposes a kinematic constraint on the discontinuity kinematics. Application of the resulting kinematic condition to an inelastic material model, e.g., a unified elastoplastic damage model considered in this section, naturally yields all the ingredients characterizing the discontinuity (band), i.e., localized constitutive laws, traction-based failure criterion and discontinuity orientation, etc.

\subsection{A unified elastoplastic damage framework}

To analyze strain localization in general inelastic softening solids, a unified elastoplastic damage framework [36, 80] is presented in this section. Both stress- and traction-based elastoplastic damage models can be developed within this framework.

\subsubsection{Stress-strain relations}

To account for both damage evolution and plastic flows, the constitutive relations are described by the following elastoplastic damage model

$\sigma=\mathbb{E}:\left(\epsilon-\epsilon^{\mathrm{p}}\right), \quad \epsilon=\mathbb{C}: \sigma+\epsilon^{\mathrm{p}}$

where the fourth-order tensors $\mathbb{E}$ and $\mathbb{C}=\mathbb{E}^{-1}$ denote the (variable) material stiffness and compliance, respectively; $\epsilon^{\mathrm{p}}$ represents the irreversible plastic strain tensor. Note that both the compliance $\mathbb{C}$ (or the stiffness $\mathbb{E}$ ) and the plastic strain $\epsilon^{\mathrm{p}}$ are internal variables.

By time differentiation, it follows that

$\dot{\sigma}=\mathbb{E}:\left(\dot{\boldsymbol{\epsilon}}-\dot{\boldsymbol{\epsilon}}^{\mathrm{dis}}\right), \quad \dot{\boldsymbol{\epsilon}}=\mathbb{C}: \dot{\boldsymbol{\sigma}}+\dot{\boldsymbol{\epsilon}}^{\mathrm{dis}}$

where the dissipative strain rate $\dot{\boldsymbol{\epsilon}}^{\text {dis }}$ is defined as $\dot{\boldsymbol{\epsilon}}^{\mathrm{dis}}:=\dot{\mathbb{C}}: \sigma+\dot{\boldsymbol{\epsilon}}^{\mathrm{p}}$, with $\dot{\mathbb{C}}: \boldsymbol{\sigma}$ and $\dot{\boldsymbol{\epsilon}}^{\mathrm{p}}$ being its damage and plastic components, respectively; see Fig. 5. Note that the dissipative strain rate $\dot{\boldsymbol{\epsilon}}^{\text {dis }}$ does not correspond to an actual "strain". It is only defined in rate form when the involved energy dissipation mechanisms, i.e., damage evolution and plastic flows, are active.

Besides the above constitutive relations, the evolution laws for the compliance tensor $\mathbb{C}$ (or the damage one $\mathbb{C}^{\mathrm{d}}$ ) and strain-like internal variables $\left\{\epsilon^{\mathrm{p}}, \kappa\right\}$ have to be postulated.

Remark 4.1 As shown in Fig. 6, the strain tensor $\epsilon$ can also be rewritten as the kinematic decomposition (3.45), in which the elastic and inelastic strain tensors $\left(\boldsymbol{\epsilon}^{\mathrm{e}}, \boldsymbol{\epsilon}^{\mathrm{in}}\right)$ are expressed as

$\epsilon^{\mathrm{e}}=\mathbb{C}^{0}: \sigma, \quad \epsilon^{\mathrm{in}}=\epsilon^{\mathrm{d}}+\epsilon^{\mathrm{p}}=\mathbb{C}^{\mathrm{d}}: \sigma+\epsilon^{\mathrm{p}}$

where the damage strain tensor $\epsilon^{\mathrm{d}}:=\mathbb{C}^{\mathrm{d}}: \sigma$ represents the recoverable inelastic strain; the fourth-order damage compliance $\mathbb{C}^{\mathrm{d}}$ is defined as $\mathbb{C}^{\mathrm{d}}:=\mathbb{C}-\mathbb{C}^{0}$, with identical evolution law $\dot{\mathbb{C}}^{\mathrm{d}}=\dot{\mathbb{C}}$. 


\subsubsection{Evolution laws}

Let us consider a rate-independent inelastic solid characterized by the following convex, smooth and differentiable failure criterion $\mathcal{F}(\sigma, q) \leq 0$, where the failure function $\mathcal{F}(\sigma, q)$ is homogeneous of degree $M \geq 1$, i.e.,

$\mathcal{F}(\boldsymbol{\sigma}, q)=\frac{1}{M}\left(\partial_{\boldsymbol{\sigma}} \mathcal{F}: \boldsymbol{\sigma}+\partial_{q} \mathcal{F} \cdot q\right)=\frac{1}{M}(\boldsymbol{\Lambda}: \boldsymbol{\sigma}-h \cdot q) \leq 0$

for the derivatives $\boldsymbol{\Lambda}:=\partial \mathcal{F} / \partial \boldsymbol{\sigma}$ and $h:=-\partial \mathcal{F} / \partial q$. Here, the stress-like internal variable $q(\kappa)$ characterizes the softening behavior of the material, with $\kappa$ being the conjugate strain-like one. The homogeneous failure function (4.4), frequently encountered in practice, automatically guarantees non-negative energy dissipation for any softening law $q(\kappa)$; see $\mathrm{Wu}$ and Cervera [79] for more details.

Similarly to the cohesive models for strong/regularized discontinuities, the following associated evolution laws are derived from the postulate of maximum energy dissipation [67] as

$\dot{\boldsymbol{\epsilon}}^{\mathrm{dis}}=\lambda \boldsymbol{\Lambda}, \quad \dot{\kappa}=\lambda h$

where the Lagrangian multiplier $\lambda$ satisfies the Kuhn-Tucker loading/unloading conditions

$\lambda \geq 0, \quad \mathcal{F}(\sigma, q) \leq 0, \quad \lambda \mathcal{F}(\sigma, q)=0$

To differentiate the damage and plastic contributions to the dissipative strain rate $\dot{\boldsymbol{\epsilon}}^{\text {dis }}$, a material parameter $\xi \in[0,1]$ is introduced so that $[36,53,80]$

$\dot{\boldsymbol{\epsilon}}^{\mathrm{p}}=(1-\xi) \dot{\boldsymbol{\epsilon}}^{\mathrm{dis}}=(1-\xi) \lambda \boldsymbol{\Lambda}$

$\dot{\mathbb{C}}: \sigma=\xi \dot{\boldsymbol{\epsilon}}^{\mathrm{dis}}=\xi \lambda \boldsymbol{\Lambda}$

The cases $\xi=0$ and $\xi=1$ correspond to the classical plasticity model and elastic damage model [10], respectively. For the intermediate value $\xi \in(0,1)$, both the material compliance $\mathbb{C}$ (or the damage one $\mathbb{C}^{\mathrm{d}}$ ) and the plastic strain $\epsilon^{\mathrm{p}}$ are internal variables, resulting in a combined plastic-damage model.

The principle of maximum dissipation determines only the components of the compliance $\mathbb{C}$ appearing in the product $\sigma: \dot{\mathbb{C}}: \sigma$, leaving the remaining ones undefined. A particular evolution law for the compliance $\mathbb{C}$ (or the damage one $\mathbb{C}^{\mathrm{d}}$ ) satisfying Eq. (4.7b) is given by $[36,80]$

$\dot{\mathbb{C}}=\dot{\mathbb{C}}^{\mathrm{d}}=\xi \lambda \frac{\boldsymbol{\Lambda} \otimes \boldsymbol{\Lambda}}{\boldsymbol{\Lambda}: \boldsymbol{\sigma}}$

as long as the condition $\boldsymbol{\Lambda}: \boldsymbol{\sigma} \neq 0$ is satisfied.

\subsubsection{Rate constitutive relations}

When the material is unloading, i.e., $\mathcal{F}(\sigma, q)<0$, it follows that $\lambda=0$; for the loading case, it follows that $\mathcal{F}(\sigma, q)=0$ and $\lambda>0$ is solved from the consistency condition $\dot{\mathcal{F}}(\sigma, q)=0$, i.e.,

$\lambda=\frac{\boldsymbol{\Lambda}: \mathbb{E}: \dot{\boldsymbol{\epsilon}}}{\boldsymbol{\Lambda}: \mathbb{E}: \boldsymbol{\Lambda}+h \cdot H \cdot h}=\frac{\boldsymbol{\Lambda}: \dot{\boldsymbol{\sigma}}}{h \cdot H \cdot h}$ 
Therefore, the rate constitutive relations are given by

$$
\begin{aligned}
\dot{\boldsymbol{\sigma}} & =\mathbb{E}:(\dot{\boldsymbol{\epsilon}}-\lambda \boldsymbol{\Lambda})=\mathbb{E}_{\mathrm{tan}}: \dot{\boldsymbol{\epsilon}} \\
\dot{\boldsymbol{\epsilon}} & =\mathbb{C}: \dot{\boldsymbol{\sigma}}+\lambda \boldsymbol{\Lambda}=\mathbb{C}_{\mathrm{tan}}: \dot{\boldsymbol{\sigma}}
\end{aligned}
$$

where the tangent stiffness and compliance are expressed as

$$
\begin{aligned}
& \mathbb{E}_{\tan }=\mathbb{E}-\frac{\mathbb{E}:(\boldsymbol{\Lambda} \otimes \boldsymbol{\Lambda}): \mathbb{E}}{\boldsymbol{\Lambda}: \mathbb{E}: \boldsymbol{\Lambda}+h \cdot H \cdot h} \\
& \mathbb{C}_{\mathrm{tan}}=\mathbb{C}+\frac{\boldsymbol{\Lambda} \otimes \boldsymbol{\Lambda}}{h \cdot H \cdot h}
\end{aligned}
$$

for the loading state (i.e., $\lambda>0$ ), both being symmetric due to the associated evolution laws assumed.

\subsection{Strain localization analysis}

For the strong discontinuity with a vanishing band width $b \rightarrow 0$, stress boundedness requires cancellation of the unbounded strain jump [15, 45-47], i.e.,

$$
\llbracket \boldsymbol{\epsilon} \rrbracket=\boldsymbol{\epsilon}_{\mathcal{S}}^{\mathrm{in}}=(\boldsymbol{e} \otimes \boldsymbol{n})^{\mathrm{sym}}=\frac{1}{b}(\boldsymbol{w} \otimes \boldsymbol{n})^{\mathrm{sym}}
$$

This is equivalent to satisfaction of the stress continuity condition (3.5). As the stresses should not depend on the band width $b$, even if the deformation vector $\boldsymbol{e}$ and the strain $\boldsymbol{\epsilon}$ do, the above arguments also hold for the regularized discontinuity with a finite band width $b \nrightarrow 0$. Note that both the stress continuity condition (3.5) and the equivalent kinematic constraint (4.12) can be written in rate form [15], but no additional insight in the problem is gained.

The kinematic constraint (4.12) states that, if strain localization can occur with a continuous stress field, difference in the strains between interior and exterior points of the discontinuity (band), i.e., the strain jump characterized by Maxwell's compatibility condition, has to be completely inelastic. This conclusion is consistent with the methodology employed in Section 3.

\subsubsection{Kinematic constraint}

Let us now consider strain localization in an inelastic solid characterized by the above elastoplastic damage model. To this end, the kinematic constraint (4.12) has to be accounted for appropriately. More specifically, upon strain localization the dissipative flow tensor characterizing the evolution laws evolves into a particular structure expressed uniquely in terms of a dissipative flow vector and the discontinuity orientation. That is, the flow tensorial components in the directions orthogonal to the discontinuity orientation have to vanish. This property allows introducing a tractionbased failure criterion and developing a localized plastic-damage model for the discontinuity (band).

Recalling the inelastic strain $(4.3)_{2}$, the kinematic condition (4.12) particularizes into

$\boldsymbol{\epsilon}^{\mathrm{in}}=(\boldsymbol{e} \otimes \boldsymbol{n})^{\mathrm{sym}}=\boldsymbol{\epsilon}^{\mathrm{d}}+\boldsymbol{\epsilon}^{\mathrm{p}}=\mathbb{C}^{\mathrm{d}}: \boldsymbol{\sigma}+\boldsymbol{\epsilon}^{\mathrm{p}}$

so that the stress continuity condition (3.5) is fulfilled upon strain localization. 
In the right hand side of the relation (4.13), the damage/plastic strains $\left(\epsilon^{\mathrm{d}}, \epsilon^{\mathrm{p}}\right)$ correspond to the recoverable and irreversible components of the inelastic strain $\epsilon^{\text {in }}$, respectively. Therefore, the (apparent) displacement jump $\boldsymbol{w}$ and the inelastic deformation vector $\boldsymbol{e}:=\boldsymbol{w} / b$ have to admit similar decompositions

$\boldsymbol{w}=\boldsymbol{w}^{\mathrm{d}}+\boldsymbol{w}^{\mathrm{p}}, \quad \boldsymbol{e}=\boldsymbol{e}^{\mathrm{d}}+\boldsymbol{e}^{\mathrm{p}}$

such that

$\left(\boldsymbol{e}^{\mathrm{p}} \otimes \boldsymbol{n}\right)^{\mathrm{sym}}=\boldsymbol{\epsilon}^{\mathrm{p}}$

$\left(e^{\mathrm{d}} \otimes n\right)^{\mathrm{sym}}=\boldsymbol{\epsilon}^{\mathrm{d}}=\mathbb{C}^{\mathrm{d}}: \sigma$

where the damage and plastic deformation vectors, $\boldsymbol{e}^{\mathrm{d}}:=\boldsymbol{w}^{\mathrm{d}} / b$ and $\boldsymbol{e}^{\mathrm{p}}:=\boldsymbol{w}^{\mathrm{d}} / b$, are defined as the recoverable and unrecoverable displacement jumps $\left(\boldsymbol{w}^{\mathrm{d}}, \boldsymbol{w}^{\mathrm{p}}\right)$ normalized with respect to the band width $b$.

It follows from Eqs. (4.7a) and (4.15a) that

$\left(\dot{\boldsymbol{e}}^{\mathrm{p}} \otimes \boldsymbol{n}\right)^{\mathrm{sym}}=\dot{\boldsymbol{\epsilon}}^{\mathrm{p}}=(1-\xi) \lambda \boldsymbol{\Lambda}$

Satisfaction of the above relation implies the existence of a dissipative flow vector $\gamma$ satisfying

$\dot{\boldsymbol{e}}^{\mathrm{p}}=(1-\xi) \lambda \boldsymbol{\gamma}, \quad(\boldsymbol{\gamma} \otimes \boldsymbol{n})^{\mathrm{sym}}=\boldsymbol{\Lambda}$

Furthermore, owing to the static constraint (3.6), the following identity holds

$\boldsymbol{\Lambda}: \sigma=\gamma \cdot(\sigma \cdot n)=\gamma \cdot t$

between the dissipative flow tensor $\boldsymbol{\Lambda}$ and vector $\boldsymbol{\gamma}$. This relation is useful to link the stress- and traction-based failure criteria characterizing the discontinuity (band).

Pre-multiplying relation $(4.17)_{2}$ by the symmetric fourth-order identity tensor $\mathbb{I}$ and then by the orientation $\boldsymbol{n}$, it follows that [47]

$\boldsymbol{\gamma}=2 \boldsymbol{n} \cdot \boldsymbol{\Lambda}-\boldsymbol{n} \Lambda_{n n}=\gamma_{n} \boldsymbol{n}+\gamma_{m} \boldsymbol{m}+\gamma_{p} \boldsymbol{p}$

where the local components $\left(\gamma_{n}, \gamma_{m}, \gamma_{p}\right)$ of the dissipative flow vector $\gamma$ are expressed as

$\gamma_{n}:=\boldsymbol{\gamma} \cdot \boldsymbol{n}=\Lambda_{n n}, \quad \gamma_{m}:=\boldsymbol{\gamma} \cdot \boldsymbol{m}=2 \Lambda_{n m}, \quad \gamma_{p}:=\boldsymbol{\gamma} \cdot \boldsymbol{p}=2 \Lambda_{n p}$

Substitution of the above dissipative flow vector $\gamma$ into Eq. $(4.17)_{2}$ yields

$\boldsymbol{\Lambda}=(\boldsymbol{\gamma} \otimes \boldsymbol{n})^{\mathrm{sym}}=\Lambda_{n n}(\boldsymbol{n} \otimes \boldsymbol{n})+2 \Lambda_{n m}(\boldsymbol{n} \otimes \boldsymbol{m})^{\mathrm{sym}}+2 \Lambda_{n p}(\boldsymbol{n} \otimes \boldsymbol{p})^{\mathrm{sym}}$

or, equivalently,

$\Lambda_{m m}\left(\boldsymbol{\theta}^{\mathrm{cr}}\right)=0, \quad \Lambda_{p p}\left(\boldsymbol{\theta}^{\mathrm{cr}}\right)=0, \quad \Lambda_{m p}\left(\boldsymbol{\theta}^{\mathrm{cr}}\right)=0$ 
where $\theta^{\text {cr }}$ denote the critical angles of the discontinuity (band).

It is concluded from Eq. (4.21) that, upon strain localization the failure criterion $\mathcal{F}(\sigma, q) \leq 0$ does not depend on the stress components $\sigma_{m m}, \sigma_{p p}$ and $\sigma_{m p}$, but is only a function of the tractions $\boldsymbol{t}:=\boldsymbol{\sigma} \cdot \boldsymbol{n}=\left\{\sigma_{n n}, \sigma_{n m}, \sigma_{n p}\right\}^{\mathrm{T}}$. Therefore, as long as the critical angles $\boldsymbol{\theta}^{\text {cr }}$ satisfying the kinematic constraints (4.21) exist, it is always possible to introduce an appropriate traction-based failure criterion to characterize the discontinuity (band); see Section 4.3.

\subsubsection{Localized constitutive model for the discontinuity (band)}

Owing to the particular structure (4.20) of the dissipative flow tensor $\boldsymbol{\Lambda}$ upon strain localization, the evolution laws (4.5) and (4.7) are naturally projected to the orientation $\boldsymbol{n}\left(\boldsymbol{\theta}^{\text {cr }}\right)$. Accordingly, the damage evolution law (4.7b) can be rewritten as

$\dot{\mathbb{C}}=\dot{\mathbb{C}}^{\mathrm{d}}=\left(\dot{C}^{\mathrm{d}} \underline{\bar{\otimes}} N\right)^{\text {sym }} \quad \Longrightarrow \quad \mathbb{C}^{\mathrm{d}}=\left(C^{\mathrm{d}} \underline{\bar{\otimes}} N\right)^{\text {sym }}$

where $\boldsymbol{N}:=\boldsymbol{n} \otimes \boldsymbol{n}$ is a second-order geometric tensor; $\boldsymbol{C}^{\mathrm{d}}$ denotes the second-order compliance tensor of the discontinuity (band), with the following evolution law

$\dot{C}^{\mathrm{d}}=\xi \lambda \frac{\boldsymbol{\gamma} \otimes \gamma}{\boldsymbol{\gamma} \cdot \boldsymbol{t}}$

In other words, upon strain localization the damage behavior of the material is sufficiently characterized by a secondorder internal variable $\boldsymbol{C}^{\mathrm{d}}$ rather than the original fourth-order one $\mathbb{C}^{\mathrm{d}}$; see Remark 4.2 for details.

Accordingly, the damage strain tensor $(4.15 b)$ becomes

$\boldsymbol{\epsilon}^{\mathrm{d}}=\left(\boldsymbol{e}^{\mathrm{d}} \otimes \boldsymbol{n}\right)^{\mathrm{sym}}=\mathbb{C}^{\mathrm{d}}: \boldsymbol{\sigma}=\left[\left(\boldsymbol{C}^{\mathrm{d}} \cdot \boldsymbol{t}\right) \otimes \boldsymbol{n}\right]^{\mathrm{sym}}$

where the static constraint (3.6) has been considered. That is, the discontinuity (band) can be described by the following localized plastic-damage constitutive relations

$\boldsymbol{e}^{\mathrm{d}}=\boldsymbol{e}-\boldsymbol{e}^{\mathrm{p}}=\boldsymbol{C}^{\mathrm{d}} \cdot \boldsymbol{t}, \quad \boldsymbol{t}=\boldsymbol{E}^{\mathrm{d}} \cdot \boldsymbol{e}^{\mathrm{d}}=\boldsymbol{E}^{\mathrm{d}} \cdot\left(\boldsymbol{e}-\boldsymbol{e}^{\mathrm{p}}\right)$

for the second-order stiffness tensor $\boldsymbol{E}^{\mathrm{d}}:=\left(\boldsymbol{C}^{\mathrm{d}}\right)^{-1}$ and the localized plastic evolution law (4.17).

By time differentiation and calling for the consistency condition, the localized discontinuity model of identical forms as in those in Table 1 can be derived. The details are omitted here. As can be seen, as long as strain localization occurs with a continuous stress field, the discontinuity (band) can be characterized by a localized model which is formally identical but not necessarily equivalent to that in the strong/regularized discontinuity approaches.

Remark 4.2 With the damage compliance tensor $(4.22)_{2}$, the material compliance tensor $\mathbb{C}$ is given by

$\mathbb{C}=\mathbb{C}^{0}+\mathbb{C}^{\mathrm{d}}=\mathbb{C}^{0}+\left(\boldsymbol{C}^{\mathrm{d}} \underline{\bar{\otimes}} N\right)^{\mathrm{sym}}$

Inversely, the material stiffness tensor $\mathbb{E}$ is obtained from the Sherman-Morrison-Woodburg formula [23]

$\mathbb{E}=\mathbb{C}^{-1}=\mathbb{E}^{0}-\mathbb{E}^{0}:\left[\left(\boldsymbol{E}^{\mathrm{d}}+\boldsymbol{n} \cdot \mathbb{E}^{0} \cdot \boldsymbol{n}\right)^{-1} \underline{\bar{\otimes}} \boldsymbol{N}\right]^{\mathrm{sym}}: \mathbb{E}^{0}$

Note the similarity between above overall material stiffness/compliance tensors and the tangent ones, i.e., Eqs. (3.41) and (3.44), obtained in the embedded/smeared discontinuity models in Section 3.4. 


\subsection{Failure criterion}

Dependent on the strategies dealing with the discontinuity orientation and the associated traction-based failure criterion, two dual approaches, not necessarily equivalent, can be developed.

\subsubsection{Traction-based elastoplastic damage model}

Provided the characteristic angles $\boldsymbol{\theta}^{\mathrm{cr}}$ of the discontinuity (band) are known a priori, say, $\boldsymbol{\theta}^{\mathrm{cr}}=\hat{\boldsymbol{\theta}}^{\mathrm{cr}}$, an explicit traction-based failure function can be introduced as in Section 3.2, i.e.,

$\mathcal{F}(\boldsymbol{\sigma}, q)=\widehat{f}(\boldsymbol{\sigma} \cdot \boldsymbol{n}, q)=\widehat{f}(\boldsymbol{t}, q) \leq 0$

Accordingly, the dissipative flow tensor $\boldsymbol{\Lambda}:=\partial \mathcal{F} / \partial \boldsymbol{\sigma}$ is evaluated as the form (4.20)

$$
\boldsymbol{\Lambda}:=(\boldsymbol{\gamma} \otimes \boldsymbol{n})^{\mathrm{sym}}, \quad \boldsymbol{\gamma}=\hat{\boldsymbol{\gamma}}:=\frac{\partial \hat{f}}{\partial \boldsymbol{t}}
$$

where the Cartesian components of the third-order tensor $\mathcal{N}=\partial \boldsymbol{t} / \partial \boldsymbol{\sigma}$ is expressed as $\mathcal{N}_{i j k}=\frac{1}{2}\left(\delta_{i j} n_{k}+\delta_{i k} n_{j}\right)$ for the Kronecker-delta $\delta_{i j}$ and the components $n_{k}$ of the normal vector $\boldsymbol{n}$.

For the dissipative flow tensor (4.28), the constitutive laws presented in Section 4.1 particularizes to the tractionbased anisotropic elastoplastic damage model suggested in $\mathrm{Wu}$ and $\mathrm{Xu}$ [80]. In this localized model, the kinematic constraint (4.12) holds for any arbitrary orientation $\boldsymbol{n}\left(\hat{\boldsymbol{\theta}}^{\mathrm{cr}}\right)$ so that the stress continuity condition (3.5) is always guaranteed during the whole failure process, i.e.,

$\boldsymbol{\Lambda}:=(\hat{\boldsymbol{\gamma}} \otimes \boldsymbol{n})^{\mathrm{sym}} \quad \Longrightarrow \quad \llbracket \boldsymbol{\sigma} \rrbracket=0$

In other words, it is implicitly assumed that strain localization can occur with a continuous stress field. This approach coincides with the first methodology upon which the strong/regularized and embedded/smeared discontinuity approaches are based. Similarly, the discontinuity orientation $\boldsymbol{n}\left(\hat{\boldsymbol{\theta}}^{\mathrm{cr}}\right)$ cannot be determined uniquely based on the given traction-based failure criterion $\hat{f}(\boldsymbol{t}, q) \leq 0$, unless extra auxiliary conditions are introduced, e.g., the Mohr's maximization postulate as in Section 3.2.

With the dissipative flow tensor (4.28), it follows from the Sherman-Morrison-Woodburg formula [23] that the tangent stiffness tensors $\mathbb{E}^{\tan }$ obtained in Eqs. (3.41) and (4.11a) are identical. Similarly, calling for the material compliance (4.26a), it can be also concluded that the tangent compliance tensors $\mathbb{C}^{\text {tan }}$ given in Eqs. (3.44) and (4.11b) coincide. Therefore, though they are developed based on different methodologies, the embedded/smeared discontinuity models are equivalent to the elastoplastic damage model, provided an identical traction-based failure criterion is employed in both methods.

Remark 4.3 Owning to the relation (4.18), the given failure criterion $\hat{f}(\boldsymbol{t}, q) \leq 0$ can be rewritten as

$F(\boldsymbol{\sigma}, q):=\hat{f}(\boldsymbol{t}, q)=\frac{1}{M}(\hat{\boldsymbol{\gamma}} \cdot \boldsymbol{t}-\hat{h} \cdot q)=\frac{1}{M}(\boldsymbol{\Lambda}: \boldsymbol{\sigma}-\hat{h} \cdot q) \leq 0$

That is, once the discontinuity orientation $\boldsymbol{n}\left(\widehat{\boldsymbol{\theta}}^{\mathrm{cr}}\right)$ is known, the given traction-based failure criterion $\hat{f}(\boldsymbol{t}, q) \leq 0$ can be equivalently expressed as a stress-based counterpart $F(\sigma, q) \leq 0$. 


\subsubsection{Stress-based projected discontinuity model}

Alternatively, let us consider a failure criterion $\mathcal{F}(\sigma, q) \leq 0$ expressed in terms of stress invariants and is then projected to the yet-unknown discontinuity orientation $\boldsymbol{n}\left(\boldsymbol{\theta}^{\mathrm{cr}}\right)$. Let us introduce the following stress-based failure function

$\mathcal{F}(\sigma, q):=\hat{F}(\mathcal{I}, q) \leq 0$

so that the dissipative flow tensor $\boldsymbol{\Lambda}$ is given by

$\boldsymbol{\Lambda}=\hat{\boldsymbol{\Lambda}}:=\frac{\partial \hat{F}}{\partial \boldsymbol{\sigma}}=\frac{\partial \hat{F}}{\partial \sigma_{1}} \boldsymbol{v}_{1} \otimes \boldsymbol{v}_{1}+\frac{\partial \hat{F}}{\partial I_{1}} \boldsymbol{I}+\frac{\partial \hat{F}}{\partial J_{2}} \boldsymbol{s}+\cdots$

where $\mathcal{I}:=\left\{\sigma_{1}, I_{1}, J_{2}\right\}$ collects the independent stress invariants, e.g., the major principal stress $\sigma_{1}$, the first invariant $I_{1}:=\operatorname{tr}(\sigma)$ of the stress tensor $\sigma$ and the second invariant $J_{2}:=\frac{1}{2} s: s$ of the deviatoric stress tensor $s:=$ $\sigma-\frac{1}{3} \operatorname{tr}(\boldsymbol{\sigma}) \boldsymbol{I}$, etc.

Accordingly, the kinematic constraint (4.20) becomes

$(\boldsymbol{\gamma} \otimes \boldsymbol{n})^{\mathrm{sym}}=\boldsymbol{\Lambda}, \quad \boldsymbol{\Lambda}=\hat{\boldsymbol{\Lambda}}:=\frac{\partial \hat{F}}{\partial \boldsymbol{\sigma}}$

That is, if and only if the discontinuity orientation $\boldsymbol{n}\left(\boldsymbol{\theta}^{\mathrm{cr}}\right)$ and the associated dissipative flow vector $\boldsymbol{\gamma}$ satisfy the kinematic constraint (4.33) for the given dissipative flow tensor $\hat{\boldsymbol{\Lambda}}$, i.e.,

$\llbracket \sigma \rrbracket=0 \quad \Longleftrightarrow \quad \hat{\Lambda}=(\gamma \otimes n)^{\mathrm{sym}}$

can strain localization occur with a continuous stress field, and vice versa.

In this case, the discontinuity orientation $\boldsymbol{n}\left(\boldsymbol{\theta}^{\mathrm{cr}}\right)$ cannot be assumed arbitrarily as in the first approach. But rather, it has to be determined consistently from the constraints (4.21) for the given stress-based dissipative flow tensor $\boldsymbol{\Lambda}=\hat{\boldsymbol{\Lambda}}$. On the one hand, as the resulting set of equations are nonlinear, the solution may not exist at all. That is, strain localization may not occur with a continuous stress field. On the other hand, provided the solution exists, it depends only on the given failure criterion and the stress state, but not on the elastic properties (i.e., Poisson's ratio).

Once the discontinuity orientation $\boldsymbol{n}\left(\boldsymbol{\theta}^{\text {cr }}\right)$ is so determined, the associated dissipative flow vector $\boldsymbol{\gamma}$ can be obtained from Eqs. (4.19a) and (4.19b). Furthermore, as the relation (4.18) holds for the given dissipative flow tensor $\boldsymbol{\Lambda}=\hat{\boldsymbol{\Lambda}}$, the projected traction-based failure criterion $f(\boldsymbol{t}, q) \leq 0$ can be determined as

$f(\boldsymbol{t}, q):=\hat{F}(\boldsymbol{\sigma}, q)=\frac{1}{M}(\hat{\boldsymbol{\Lambda}}: \boldsymbol{\sigma}-\hat{h} \cdot q)=\frac{1}{M}(\boldsymbol{\gamma} \cdot \boldsymbol{t}-\hat{h} \cdot q) \leq 0$

Note that the above traction-based failure function $f(\boldsymbol{t}, q) \leq 0$, projected from the given stress-based one $\widehat{F}(\boldsymbol{\sigma}, q) \leq$ 0 , is not necessarily identical to the counterpart $\hat{f}(\boldsymbol{t}, q) \leq 0$ explicitly assumed $a$ priori.

In summary, provided the kinematic constant resulting from the stress continuity condition is fulfilled, consistent localized constitutive relations for the discontinuity (band) naturally emerge from the strain localization analysis of stress-based models with regularized softening. 


\section{Comparisons and discussion}

Though different methodologies are employed, both the traction-based embedded/smeared discontinuity models (or the equivalent elastoplastic damage model) and the stress-based projected discontinuity model can be developed within the unified framework presented in in Section 4. It is worthwhile to investigate the bi-directional connections between these two families of models, in particular, the conditions upon which they are equivalent.

\subsection{Evolution laws}

In both families of models, the inelastic behavior, completely localized within the discontinuity (band), is characterized by a dissipative flow tensor $\boldsymbol{\Lambda}$, expressed as the unified form (4.20) in terms of the dissipative flow vector $\boldsymbol{\gamma}$ and the orientation $\boldsymbol{n}$; see Eqs. (4.28) and (4.33), respectively. However, the physical interpretations are different.

For the traction-based embedded/smeared discontinuity models, the inelastic flow vector is given as $\boldsymbol{\gamma}=\hat{\gamma}:=$ $\partial \widehat{f} / \partial \boldsymbol{t}$. In this case, the discontinuity orientation $\boldsymbol{n}\left(\hat{\boldsymbol{\theta}}^{\mathrm{cr}}\right)$ can be assumed arbitrarily. That is, the relation (4.28) imposes no kinematic constraint. But rather, it acts only as a definition, from which the dissipative flow tensor $\boldsymbol{\Lambda}$ is determined straightforwardly in such a way that the stress continuity (3.5) is always satisfied a priori upon strain localization.

Comparatively, in the stress-based projected discontinuity model both the dissipative flow vector $\boldsymbol{\gamma}$ and the orientation $\boldsymbol{n}\left(\boldsymbol{\theta}^{\mathrm{cr}}\right)$ are unknown and even might not exist at all. In this case, the relation (4.33) imposes necessary and sufficient kinematic constraint on the occurrence of strain localization with a continuous stress field. Accordingly, the discontinuity orientation $\boldsymbol{n}\left(\widehat{\boldsymbol{\theta}}^{\mathrm{cr}}\right)$ can only be determined from the kinematic constraints (4.21) for the given dissipative flow tensor $\boldsymbol{\Lambda}=\hat{\boldsymbol{\Lambda}}:=\partial \hat{f} / \partial \boldsymbol{\sigma}$. If such discontinuity orientation exists, the dissipative flow tensor $\boldsymbol{\gamma}$ is given from the relations (4.19). Otherwise, strain localization cannot occur with stress continuity.

In summary, even if the dissipative flow vectors coincide in both families of models, the resulting evolution laws, characterized by the dissipative flow tensor (4.20), are not necessarily identical, since the discontinuity orientations might be different. An interesting question then naturally arises: whether and when would the discontinuity orientations in both families of models coincide?

\subsection{Orientation of the discontinuity (band)}

As mentioned before, for the embedded/smeared discontinuity models the orientation $\boldsymbol{n}\left(\hat{\boldsymbol{\theta}}^{\mathrm{cr}}\right)$ cannot be determined uniquely from the given traction-based failure criterion $\widehat{f}(\boldsymbol{t}, q) \leq 0$. After recalling the relations (4.19b), the stationary condition (3.9) of the Mohr's maximization postulate can be rewritten as

$$
\left.\frac{\partial \hat{f}}{\partial \boldsymbol{\theta}}\right|_{\widehat{\boldsymbol{\theta}}^{\mathrm{cr}}}=-\left(\Lambda_{m m} \frac{\partial \sigma_{m m}}{\partial \boldsymbol{\theta}}+2 \Lambda_{m p} \frac{\partial \sigma_{m p}}{\partial \boldsymbol{\theta}}+\Lambda_{p p} \frac{\partial \sigma_{p p}}{\partial \boldsymbol{\theta}}\right)_{\widehat{\boldsymbol{\theta}}^{\mathrm{cr}}}=\boldsymbol{\theta}
$$

where the identity $\boldsymbol{\Lambda}:(\partial \boldsymbol{\sigma} / \partial \boldsymbol{\theta})=0$, resulting from the coaxial property between the tensors $\boldsymbol{\Lambda}:=\partial \hat{f} / \partial \boldsymbol{\sigma}$ and $\boldsymbol{\sigma}$, has been considered. As the failure function $\hat{f}(\boldsymbol{t}, q) \leq 0$ depends only on the tractions $\boldsymbol{t}:=\left\{\sigma_{n n}, \sigma_{n m}, \sigma_{n p}\right\}^{\mathrm{T}}$, the condition (5.1) is fulfilled for arbitrary values of the remaining stress components $\left(\sigma_{m m}, \sigma_{m p}, \sigma_{p p}\right)$. This fact yields $\Lambda_{m m}\left(\hat{\boldsymbol{\theta}}^{\mathrm{cr}}\right)=0, \quad \Lambda_{p p}\left(\hat{\boldsymbol{\theta}}^{\mathrm{cr}}\right)=0, \quad \Lambda_{m p}\left(\hat{\boldsymbol{\theta}}^{\mathrm{cr}}\right)=0$ 
These relations correspond exactly to the kinematic constraints (4.21). Note that the maximization condition (3.10) should be verified for the discontinuity angles $\widehat{\boldsymbol{\theta}}^{\mathrm{cr}}$ determined from Eqs. (5.2).

Inversely, in the projected discontinuity model with a given stress-based failure criterion, strain localization with a continuous stress field can occur, if and only if the critical angles $\boldsymbol{\theta}^{\text {cr }}$ satisfying the kinematic constraints (4.21) exist. Upon this condition, a traction-based failure criterion $f(\boldsymbol{t}, q) \leq 0$ can always be obtained by projecting the given stress-based one $\hat{F}(\sigma, q) \leq 0$. Accordingly, for the discontinuity (band) to form along the orientation $\boldsymbol{n}\left(\boldsymbol{\theta}^{\mathrm{cr}}\right)$, the projected traction-based failure criterion $f(t, q)=0$ is activated. Meanwhile, no discontinuity (band) forms at any other orientation $\boldsymbol{n}(\boldsymbol{\theta}) \neq \boldsymbol{n}\left(\boldsymbol{\theta}^{\mathrm{cr}}\right)$. The above facts transform into

$f[\boldsymbol{\sigma} \cdot \boldsymbol{n}(\boldsymbol{\theta}), q] \leq f\left[\boldsymbol{\sigma} \cdot \boldsymbol{n}\left(\boldsymbol{\theta}^{\mathrm{cr}}\right), q\right]=0$

That is, the tractions $\boldsymbol{\sigma} \cdot \boldsymbol{n}\left(\boldsymbol{\theta}^{\mathrm{cr}}\right)$ maximize the failure function $f(\boldsymbol{t}, q)=f[\boldsymbol{\sigma} \cdot \boldsymbol{n}(\boldsymbol{\theta}), q]$. Therefore, the discontinuity angles $\boldsymbol{\theta}^{\mathrm{cr}}$ satisfying Eqs. (4.21) coincide with those determined from Mohr's maximization postulate.

Remark 5.1 In embedded/smeared discontinuity models, it is assumed a priori that the strain localization with a continuous stress field can always occur. Therefore, if the solution to Eqs. (5.2) does not exist, the discontinuity angles $\widehat{\boldsymbol{\theta}}^{\mathrm{cr}}$ for the traction-based localized models should be determined from another set of solution to Eqs. (5.1)

$\left.\frac{\partial \sigma_{m m}}{\partial \boldsymbol{\theta}}\right|_{\widehat{\boldsymbol{\theta}}^{\mathrm{cr}}}=0,\left.\quad \frac{\partial \sigma_{m p}}{\partial \boldsymbol{\theta}}\right|_{\widehat{\boldsymbol{\theta}}^{\mathrm{cr}}}=0,\left.\quad \frac{\partial \sigma_{p p}}{\partial \boldsymbol{\theta}}\right|_{\widehat{\boldsymbol{\theta}}^{\mathrm{cr}}}=0$

Contrariwise, for the stress-based projected discontinuity model if the solution to the kinematic constraints (4.21) does not exist, strain localization upon stress continuity cannot occur. In this situation, the given stress-based failure criterion can be modified based on the solution to Eq. (5.4) in such a way that both families of models are completely equivalent; see Wu and Cervera [79] for the details.

\subsection{Equivalence conditions between traction- and stress-based localized models}

With the above arguments, it can be concluded that:

- An embedded/smeared discontinuity model is equivalent to the projected discontinuity model with a given stress-based failure criterion $\widehat{F}(\sigma, q) \leq 0$, provided the traction-based failure criterion in the former is adopted as $\hat{f}(\boldsymbol{t}, q)=f(\boldsymbol{t}, q) \leq 0$ and Mohr's maximization postulate is used to determine the discontinuity orientation. Here, the traction-based failure criterion $f(\boldsymbol{t}, q) \leq 0$ is projected from the given stress-based counterpart $\hat{F}(\boldsymbol{\sigma}, q) \leq 0$ to the orientation $\boldsymbol{n}\left(\boldsymbol{\theta}^{\mathrm{cr}}\right)$ which satisfies the kinematic constraint (4.33).

- A projected discontinuity model is equivalent to the embedded/smeared discontinuity model with a tractionbased failure criterion $\hat{f}(\boldsymbol{t}, q) \leq 0$ and the discontinuity orientation $\boldsymbol{n}\left(\hat{\boldsymbol{\theta}}^{\mathrm{cr}}\right)$ determined from Mohr's maximization postulate, if the stress-based failure criterion in the former is adopted as $\hat{F}(\boldsymbol{\sigma}, q)=\hat{f}\left[\boldsymbol{\sigma} \cdot \boldsymbol{n}\left(\hat{\boldsymbol{\theta}}^{\mathrm{cr}}\right), q\right] \leq 0$. In this case, the discontinuity orientation $\boldsymbol{n}\left(\boldsymbol{\theta}^{\text {cr }}\right)$ and the associated dissipative flow vector $\boldsymbol{\gamma}$ satisfying the kinematic constraint (4.33) coincide with the given ones $\boldsymbol{n}\left(\hat{\boldsymbol{\theta}}^{\mathrm{cr}}\right)$ and $\hat{\boldsymbol{\gamma}}:=\partial \hat{f} / \partial \boldsymbol{t}$. 
Upon the above conditions, the inelastic evolution laws coincide for both families of models, i.e.,

$\boldsymbol{\gamma}=\hat{\boldsymbol{\gamma}}, \quad \boldsymbol{n}\left(\boldsymbol{\theta}^{\mathrm{cr}}\right)=\boldsymbol{n}\left(\hat{\boldsymbol{\theta}}^{\mathrm{cr}}\right) \quad \Longleftrightarrow \quad \boldsymbol{\Lambda}=\hat{\boldsymbol{\Lambda}}$

Provided identical softening laws $q(\kappa)$ or $q(\bar{\kappa})$ are used, the traction-based embedded/smeared discontinuity models (or the equivalent elastoplastic damage model) and the stress-based projected discontinuity model are equivalent to each other.

\section{Plane stress examples}

In this section the general 3D results presented in previous sections are particularized to the plane stress condition $\left(\sigma_{3}=0\right)$. The plane strain results are discussed in Wu and Cervera [79].

As shown in Fig. 7, a 2D solid $\Omega \subset \mathbb{R}^{2}$ with a discontinuity $\mathcal{S}$ is considered. The unit normal vector $\boldsymbol{n}$ and the tangential vector $\boldsymbol{m}$ of the discontinuity $\mathcal{S}$ are on the plane of interest, with the other tangential vector $\boldsymbol{p}$ perpendicular to that plane. The in-plane principal stresses are denoted by $\sigma_{1}$ and $\sigma_{2}\left(\sigma_{1} \geq \sigma_{2}\right)$, respectively, while the third one $\sigma_{3}$ is orthogonal to that plane. In such $2 \mathrm{D}$ cases the discontinuity orientation can be characterized by the inclination angle (anti-clockwise) $\theta \in[-\pi / 2, \pi / 2]$ between the normal vector $\boldsymbol{n}$ and the principal vector $\boldsymbol{v}_{1}$ of the stress tensor.

\subsection{Explicit traction-based failure criterion}

In the case of plane stress, the stationarity condition (3.9) for a given traction-based failure criterion $\hat{f}(\boldsymbol{t}, q) \leq 0$ simplifies as

$\left.\frac{\partial \hat{f}}{\partial \theta}\right|_{\hat{\theta}^{\mathrm{cr}}}=-\left(\sigma_{1}-\sigma_{2}\right)\left[\hat{\gamma}_{n} \sin \left(2 \hat{\theta}^{\mathrm{cr}}\right)-\hat{\gamma}_{m} \cos \left(2 \hat{\theta}^{\mathrm{cr}}\right)\right]=0$

The discontinuity angle $\hat{\theta}^{\text {cr }}$ is then determined from

$\tan \left(2 \hat{\theta}^{\mathrm{cr}}\right)=\frac{\hat{\gamma}_{m}}{\hat{\gamma}_{n}} \quad$ and $\left.\quad \frac{\partial^{2} \hat{f}}{\partial \theta^{2}}\right|_{\hat{\theta}^{\mathrm{cr}}}<0$

In general, two values can be obtained. The corresponding stress-based failure criterion (4.30) becomes

$F(\sigma, q)=\frac{1}{M}\left[\left(\Lambda_{1} \sigma_{1}+\Lambda_{2} \sigma_{2}\right)-\hat{h} \cdot q\right] \leq 0$

where the relation $\Lambda_{3} \sigma_{3}=0$, fulfilled in the case of plane stress $\left(\sigma_{3}=0\right)$, has been considered.

\subsection{Projected traction-based failure criterion}

Regarding a given stress-based failure criterion $\hat{F}(\sigma, q) \leq 0$, the discontinuity angle $\theta^{\text {cr }}$ is determined from the kinematic constraints (4.21) for the dissipative flow tensor $\boldsymbol{\Lambda}=\hat{\boldsymbol{\Lambda}}:=\partial \hat{F} / \partial \boldsymbol{\sigma}$. As $\hat{\Lambda}_{p p}=0$ and $\hat{\Lambda}_{m p}=0$ are automatically (always) satisfied in the plane stress condition, it follows from the constraint $\hat{\Lambda}_{p p}\left(\theta^{\text {cr }}\right)=0$ that

$\cos \left(2 \theta^{\text {cr }}\right)=\frac{\hat{\Lambda}_{1}+\hat{\Lambda}_{2}}{\hat{\Lambda}_{1}-\hat{\Lambda}_{2}}, \quad \sin \left(2 \theta^{\text {cr }}\right)=2 \operatorname{sign}\left(\sigma_{n m}\right) \frac{\sqrt{-\hat{\Lambda}_{1} \hat{\Lambda}_{2}}}{\hat{\Lambda}_{1}-\hat{\Lambda}_{2}}$ 
if the conditions $\hat{\Lambda}_{1} \geq 0$ and $\hat{\Lambda}_{2} \leq 0$ are satisfied.

With the discontinuity angle $\theta^{\text {cr }}$ defined from Eq. (6.4), the traction-based failure criterion $f(\boldsymbol{t}, q) \leq 0$ projected from the stress-based counterpart $\widehat{F}(\sigma, q) \leq 0$ is then obtained from Eq. (4.35) as

$f(\boldsymbol{t}, q)=\frac{1}{M}\left[\left(\hat{\Lambda}_{1}+\hat{\Lambda}_{2}\right) t_{n}+2 \sqrt{-\hat{\Lambda}_{1} \hat{\Lambda}_{2}}\left|t_{m}\right|-\hat{h} \cdot q\right] \leq 0$

for the normal and tangential tractions $\left(t_{n}, t_{m}\right)$.

Remark 6.1 If the discontinuity angle $\theta^{\text {cr }}$ determined from Eq. (6.4) exists, it can be verified that, the following stationarity conditions associated with the projected traction-based failure criterion (6.5) are satisfied

$\left.\frac{\partial f}{\partial \theta}\right|_{\theta^{\text {cr }}}=0,\left.\quad \frac{\partial^{2} f}{\partial \theta^{2}}\right|_{\theta^{\text {cr }}}<0$

Namely, the tractions $\left(t_{n}, t_{m}\right)$ on the orientation $\boldsymbol{n}\left(\theta^{\mathrm{cr}}\right)$ maximize the traction-based failure criterion $f(\boldsymbol{t}, q) \leq 0$.

Remark 6.2 For the case $\hat{\Lambda}_{2}>0$ or $\hat{\Lambda}_{1}<0$, Eq. (6.4) does not hold and the projection relation (4.33) cannot be fulfilled for the given stress-based failure criterion $\hat{f}(\sigma, q) \leq 0$. As mentioned in Remark 5.1, some modifications should be made in this situation so that the discontinuity angle is determined as in the traction-based models, i.e., $\theta^{\text {cr }}=0$ if $\hat{\Lambda}_{1}>\hat{\Lambda}_{2}>0$ and $\theta^{\text {cr }}=\pi / 2$ if $\hat{\Lambda}_{2}<\hat{\Lambda}_{1}<0$; see Wu and Cervera [79] for the details.

Remark 6.3 For a softening material with associated evolution laws, in the plane stress condition the discontinuity angle determined from Eq. (6.4) coincides with that obtained from the classical discontinuous bifurcation analysis [66]. However, for the cases of non-associated evolution laws or plane strain they are in general different.

\subsection{A generic failure criterion}

In this section a generic stress-based failure criterion is considered in the plane stress condition. Depending on the involved material parameters, the failure surface defines either an ellipse, a parabola, a hyperbola or the product of two straight lines. The classical von Mises, Drucker-Prager and Mohr-Coulomb criteria are also recovered as particular examples. In all cases, the discontinuity angles and the associated traction-based failure criteria are derived explicitly.

\subsubsection{Stress-based failure criterion}

Let us consider the following stress-based failure criterion $\widehat{F}(\sigma, q) \leq 0$

$\widehat{F}(\sigma, q)=J_{2}-\frac{1}{6} A_{0} I_{1}^{2}+\frac{1}{3} B_{0} q I_{1}-C_{0} q^{2} \leq 0$

where the non-negative parameters $B_{0}$ and $C_{0}$ are related to the parameter $A_{0}<2$ through

$B_{0}=\frac{2-A_{0}}{2}(\rho-1) \geq 0, \quad C_{0}=\frac{2-A_{0}}{6} \rho \geq 0$

with the ratio $\rho:=f_{c} / f_{t} \geq 1$ between the uniaxial compressive and tensile strengths $f_{c}$ and $f_{t}$. The residual strength $q(\kappa)$ is normalized so that its initial value is $q^{0}=f_{t}$. It can be verified that the above failure criterion $\hat{F}(\sigma, q) \leq 0$ is a homogeneous function of degree $M=2$. 
In the plane stress condition $\left(\sigma_{3}=0\right)$, the failure criterion (6.7) becomes

$\widehat{F}(\sigma, q)=\frac{2-A_{0}}{6}\left(\sigma_{1}^{2}+\sigma_{2}^{2}\right)-\frac{1+A_{0}}{3} \sigma_{1} \sigma_{2}+\frac{B_{0}}{3}\left(\sigma_{1}+\sigma_{2}\right) q-C_{0} q^{2} \leq 0$

in the $\sigma_{1}-\sigma_{2}$ principal stress space. For the model parameter $A_{0}<2$ the failure surface $\hat{F}(\sigma, q)=0$ is less open than a right angle [21], which is the case interested here. Furthermore, only for the parameters satisfying $A_{0} \leq 1 / 2+B_{0}^{2} /\left(6 C_{0}\right)$ can the failure surface $\hat{F}(\sigma, q)=0$ intersect the hydrostatic axis $\sigma_{1}=\sigma_{2}$. It then follows from the relations (6.8) that the admissible parameter $A_{0}$ is within the range

$A_{0} \leq \frac{2\left(\rho^{2}-\rho+1\right)}{(\rho+1)^{2}}<2$

Accordingly, the following function types can be defined for the failure criterion (6.9)

$\begin{cases}A_{0}<\frac{1}{2} & \text { Elliptical function; see Figure 8(a) } \\ A_{0}=\frac{1}{2} & \text { Parabolic function; see Figure 9(a) } \\ \frac{1}{2}<A_{0}<2\left(\rho^{2}-\rho+1\right) /(\rho+1)^{2} & \text { Hyperbolic function; see Figure 10(a) } \\ A_{0}=2\left(\rho^{2}-\rho+1\right) /(\rho+1)^{2} & \text { Product of two straight lines; see Figure 11(a) }\end{cases}$

Remark 6.4 The classical Drucker-Prager criterion is recovered for the model parameters satisfying $A_{0}=B_{0}^{2} /\left(6 C_{0}\right)$, or, equivalently,

$A_{0}=2 \alpha^{2}, \quad B_{0}=2 \alpha(1+\alpha), \quad C_{0}=\frac{1}{3}(1+\alpha)^{2}$

for the material constant $\alpha:=(\rho-1) /(\rho+1) \in[0,1)$ dependent on the strength ratio $\rho$. In particular, von Mises criterion corresponds to $\alpha=0$.

Remark 6.5 In the plane stress condition the model parameters satisfying $B_{0}^{2}=3\left(2 A_{0}-1\right) C_{0}$ are determined as

$A_{0}=\frac{2\left(\rho^{2}-\rho+1\right)}{(\rho+1)^{2}}, \quad B_{0}=\frac{3 \rho(\rho-1)}{(\rho+1)^{2}}, \quad C_{0}=\frac{\rho^{2}}{(\rho+1)^{2}}$

Accordingly, the left branch interested coincides with the classical Mohr-Coulomb criterion, i.e.,

$\frac{\rho \sigma_{1}-\sigma_{2}-\rho q}{\rho+1}=\frac{1}{2}\left[\left(\sigma_{1}+\sigma_{2}\right) \sin \varphi+\left(\sigma_{1}-\sigma_{2}\right)-q(1+\sin \varphi)\right] \leq 0$

where the internal friction angle $\varphi \in[0, \pi / 2]$ of the material is given by

$\sin \varphi=\frac{\rho-1}{\rho+1} \quad \Longleftrightarrow \quad \rho=\frac{1+\sin \varphi}{1-\sin \varphi}$

Note that the Mohr-Coulomb criterion (6.14) applies for $\sigma_{1} \geq 0$ and $\sigma_{2} \leq 0$ in the plane stress state $\left(\sigma_{3}=0\right)$.

\subsubsection{Discontinuity angle and traction-based failure criterion}

For a discontinuity (band) to form, the failure criterion (6.9) is activated. That is, the residual strength $q \in\left[0, f_{t}\right]$ can be expressed in terms of the principal stress $\sigma_{1}$

$\widehat{F}(\sigma, q)=0 \quad \Longrightarrow \quad q=\alpha_{r} \sigma_{1}$ 
where the coefficient $\alpha_{r}(r) \in[0,1]$ depends on the stress ratio $r:=\sigma_{2} / \sigma_{1}$

$$
\begin{aligned}
& \alpha_{r}=\frac{B_{0}(1+r) \pm \sqrt{B_{0}^{2}(1+r)^{2}+6 A_{r} C_{0}}}{6 C_{0}} \\
& A_{r}=\left(2-A_{0}\right)-2\left(1+A_{0}\right) r+\left(2-A_{0}\right) r^{2}
\end{aligned}
$$

The discontinuity angle $\theta^{\text {cr }}$ is then given from Eq. (6.4) by

$$
\cos \left(2 \theta^{\text {cr }}\right)=\frac{2 B_{0} \alpha_{r}+\left(1-2 A_{0}\right)(1+r)}{3(1-r)}
$$

Clearly, it depends on the stress ratio $r:=\sigma_{2} / \sigma_{1}$ which remains fixed for a proportional load path.

For the discontinuity angle (6.18), the projected traction-based failure criterion $f(\boldsymbol{t}, q) \leq 0$, also homogeneous of degree $M=2$, is determined from Eq. (6.5) as

$f(\boldsymbol{t}, q)=t_{m}^{2}-\frac{1}{2-A_{0}}\left[\left(A_{0}-\frac{1}{2}\right) t_{n}^{2}-B_{0} q t_{n}+\frac{1}{6} B_{0}^{2} q^{2}\right]-C_{0} q^{2} \leq 0$

For the admissible parameter $A_{0} \leq 2\left(\rho^{2}-\rho+1\right) /(\rho+1)^{2}$, the following cases can be identified:

- $A_{0}<\frac{1}{2}$ : As shown in Figure 8(b), the failure criterion (6.19) defines an ellipse on the $t_{n}-t_{m}$ plane

$$
f(\boldsymbol{t}, q)=t_{m}^{2}+\frac{\frac{1}{2}-A_{0}}{2-A_{0}}\left(t_{n}+\frac{B_{0}}{1-2 A_{0}} q\right)^{2}-\left[\frac{B_{0}^{2}}{3\left(1-2 A_{0}\right)}+C_{0}\right] q^{2} \leq 0
$$

with its foci on the axis $t_{n}$ and centered at $t_{n}=-B_{0} q /\left(1-2 A_{0}\right)$. An interesting particular case corresponds to $\rho=1.0$, i.e., $B_{0}=0$ and $C_{0}=\left(2-A_{0}\right) / 6$. In this case, the failure criterion (6.20) becomes

$$
\sqrt{t_{n}^{2}+\beta^{-2} t_{m}^{2}}-\frac{2-A_{0}}{\sqrt{3\left(1-2 A_{0}\right)}} q \leq 0
$$

for the parameter $\beta:=\sqrt{\left(\frac{1}{2}-A_{0}\right) /\left(2-A_{0}\right)} \in[0,1]$. The failure criterion (6.21) is exactly the one suggested by Camacho and Ortiz [8], Pandolfi et al. [57], Jirásek and Zimmermann [29] for modeling mixed-mode failure.

- $A_{0}=\frac{1}{2}$ : As shown in Figure 9(b), the failure criterion (6.19) is a parabola on the $t_{n}-t_{m}$ plane

$$
f(\boldsymbol{t}, q)=t_{m}^{2}+\beta_{1} q t_{n}-\beta_{2} q^{2} \leq 0
$$

where the parameters $\beta_{1}$ and $\beta_{2}$ are expressed as

$$
\beta_{1}=\frac{2}{3} B_{0}=\frac{1}{2}(\rho-1), \quad \beta_{2}=\frac{1}{9} B_{0}^{2}+C_{0}=\frac{1}{16}(\rho+1)^{2}
$$

The parabolic traction-based failure criterion has been adopted in the embedded discontinuity models [39, 42].

- $\frac{1}{2}<A_{0}<2\left(\rho^{2}-\rho+1\right) /(\rho+1)^{2}$, or equivalently, $B_{0}^{2}>3\left(2 A_{0}-1\right) C_{0}$ : As shown in Figure 10(b), the failure criterion (6.19) defines a hyperbola on the $t_{n}-t_{m}$ plane, with the left branch of interest expressed as

$$
\tan \varphi \cdot t_{n}+\sqrt{t_{m}^{2}+\omega^{2} q^{2}}-c \leq 0
$$


where $\tan \varphi$ and $c$ denote the friction coefficient and cohesion of the asymptotic Mohr-Coulomb criterion

$\tan \varphi=\sqrt{\frac{A_{0}-1 / 2}{2-A_{0}}}, \quad c=\frac{B_{0} q}{\sqrt{2\left(2-A_{0}\right)\left(2 A_{0}-1\right)}}, \quad \omega=\sqrt{\frac{B_{0}^{2}}{3\left(2 A_{0}-1\right)}-C_{0}}$

Similar hyperbolic failure function has been adopted in the literature to describe the normal and shear coupling of cohesive cracks [9, 19, 40, 70, 73]; see Section 6.3 .3 for further discussion.

- $A_{0}=2\left(\rho^{2}-\rho+1\right) /(\rho+1)^{2}$, or equivalently, $B_{0}^{2}=3\left(2 A_{0}-1\right) C_{0}$ : As shown in Figure 11(b), the failure function (6.19) becomes the product of two straight lines on the $t_{n}-t_{m}$ plane, with the left branch of interest corresponding to $\omega=0$ in the hyperbolic criterion (6.24)

$\tan \varphi \cdot t_{n}+\left|t_{m}\right|-c \leq 0$

where the internal friction coefficient $\tan \varphi$ and the cohesion $c$ are given by

$\tan \varphi=\frac{1}{2} \frac{\rho-1}{\sqrt{\rho}}, \quad c=\frac{1}{2} \sqrt{\rho} q$

This is exactly the classical Mohr-Coulomb criterion.

As can be seen, the function types of the projected traction-based failure criterion $f(\boldsymbol{t}, q) \leq 0$ coincide with those of the stress-based failure criterion $\widehat{F}(\sigma, q) \leq 0$ classified in Eq. (6.11).

Remark 6.6 The traction-based failure criterion projected from the classical Drucker-Prager model can be obtained by substituting the model parameters (6.12) into Eq. (6.19), i.e.,

$f(\boldsymbol{t}, q)=t_{m}^{2}-\frac{4 \alpha^{2}-1}{4\left(1-\alpha^{2}\right)} t_{n}^{2}+\frac{\alpha}{1-\alpha} q t_{n}-\frac{1+\alpha}{3(1-\alpha)} q^{2} \leq 0$

Depending on the value of the parameter $\alpha:=\sqrt{A_{0} / 2} \in[0,1)$, the traction-based failure criterion (6.28) defines: (i) an ellipse for $0 \leq \alpha<1 / 2$ or, equivalently, $0 \leq A_{0}<1 / 2$; (ii) a parabola for $\alpha=1 / 2$ or, equivalently, $A_{0}=1 / 2$, and (iii) a hyperbola for $1 / 2<\alpha<1$ or, equivalently, $1 / 2<A_{0}<2\left(\rho^{2}-\rho+1\right) /(\rho+1)^{2}$, respectively, on the $t_{n}-t_{m}$ plane. Note that the condition $A_{0}=2\left(\rho^{2}-\rho+1\right) /(\rho+1)^{2}$, corresponding to $B_{0}^{2}=3\left(2 A_{0}-1\right) C_{0}$, cannot be reached for Drucker-Prager criterion in the case of plane stress.

\subsubsection{Further discussion on the hyperbolic failure criterion}

In the literature $[9,19,40,70,73]$, different forms of hyperbolic traction-based failure criteria have been proposed for the modeling of localized failure in quasi-brittle materials, owing to the excellent data fitting capabilities and other advantageous features (e.g., no slope discontinuity at the tip, asymptotically approaching to Mohr-Coulomb criterion for increasing compression, and a constant friction angle, etc.). However, the involved parameters are essentially mesoscopical entities hard to be determined from standard experimental tests. This shortcoming restrains heavily its application. In previous sections we have established the theoretical connections and equivalence conditions between 
localized models with traction- and stress-based failure criteria. Such a correspondence facilitates identifying those mesoscopic parameters involved in the traction-based failure criterion from the easily obtained macroscopic ones.

Let us now consider the following traction-based failure criterion [9, 73]

$\widehat{f}(\boldsymbol{t}, q)=t_{m}^{2}-\left(c-t_{n} \cdot \tan \varphi\right)^{2}+(c-\chi \cdot \tan \varphi)^{2} \leq 0$

with the left branch of interest expressed as [40]

$\tan \varphi \cdot t_{n}+\sqrt{t_{m}^{2}+(c-\chi \cdot \tan \varphi)^{2}}-c \leq 0$

where $\chi$ denotes the failure strength, not necessarily identical to the macroscopic material tensile strength $f_{t} ; \tan \varphi$ and $c$ represent the friction and cohesion of the asymptotic Mohr-Coulomb criterion. All these parameters cannot be determine easily and in general are assumed arbitrarily $[9,19,73]$.

As shown in Section 6.3.2, for the model parameter $\frac{1}{2}<A_{0}<2\left(\rho^{2}-\rho+1\right) /(\rho+1)^{2}$, in the condition of plane stress an inelastic softening solid characterized by the stress-based failure criterion (6.7) localizes into a discontinuity (band) described by the traction-based failure criterion (6.24) of identical form to Eq. (6.30). Obviously, the equivalence between them implies that

$\omega q=c-\chi \cdot \tan \varphi \quad \Longleftrightarrow \quad \chi=\frac{c-\omega q}{\tan \varphi}$

where the friction coefficient $\tan \varphi$, the cohesion $c$ and the parameter $\omega$ are given in Eq. (6.25).

Reversely, making some straightforward manipulations, the stationarity condition (6.1) for the failure criterion (6.29) yields the following discontinuity angle $\widehat{\theta}^{\text {cr }}$

$\cos \left(2 \hat{\theta}^{\mathrm{cr}}\right)=\frac{\tan \varphi}{1+\tan ^{2} \varphi} \cdot \frac{2 c-\left(\sigma_{1}+\sigma_{2}\right) \tan \varphi}{\sigma_{1}-\sigma_{2}}$

The normal and tangential tractions $\left(t_{n}, t_{m}\right)$ acting on the orientation $\boldsymbol{n}\left(\hat{\theta}^{\mathrm{cr}}\right)$ can then be expressed in terms of the principal stresses $\sigma_{1}$ and $\sigma_{2}$. Substitution of the obtained results into Eq. (6.29) yields

$F(\sigma, q)=\frac{\left(\sigma_{1}-\sigma_{2}\right)^{2}}{4}-\frac{1}{1+\tan ^{2} \varphi}\left(c-\frac{\sigma_{1}+\sigma_{2}}{2} \tan \varphi\right)^{2}+(c-\chi \cdot \tan \varphi)^{2} \leq 0$

With the relations (6.25) and (6.31), the discontinuity angle (6.18) are recovered and the reconstructed stress-based failure criterion (6.33) coincides with the given counterpart (6.9).

Therefore, a localized model with the stress-based failure criterion (6.9) is indeed equivalent to that with the given traction-based one (6.29), provided the involved parameters are consistently connected as in Eqs. (6.25) and (6.31).

As an example, let us consider a strength ratio $\rho:=f_{c} / f_{t}=10$, typical for concrete. In order for a discontinuity (band) characterized by the hyperbolic traction-based failure criterion (6.24) to form in the plane stress condition, the model parameter $A_{0}$ has to be in the range $A_{0} \in(0.5,1.504)$. As shown in Figure 12(a), the biaxial strength envelope corresponding to the following parameters

$$
A_{0}=1.45 \quad \Longrightarrow \quad B_{0}=2.475, \quad C_{0}=0.917
$$


fits the test data of normal concrete in tension-tension and tension-compression rather well. It immediately follows from the relations (6.25) and (6.31) that

$\tan \varphi=1.314, \quad c=1.712 q, \quad \chi=q$

The corresponding traction-based failure criterion is shown in Figure 12(b). Furnished with an appropriate softening law $q(\kappa)$, it can be used in the modeling of localized failure in concrete. This topic will be reported elsewhere.

\section{Conclusions}

This paper addresses traction- and stress-based approaches for the modeling of strong/regularized discontinuities induced localized failure in solids. Different approaches resulting from two complementary methodologies, i.e., discontinuities localized in an elastic solid and strain localization of an inelastic solid are systematically investigated.

In the first methodology, discontinuities are localized in elastic solids along a known orientation. It is assumed a priori that the bulk material remains elastic during the whole deformation process and all inelastic behavior is localized within the discontinuity (band). That is, strain localization always occurs with a continuous stress field. A traction-based failure criterion can be introduced to characterize the discontinuity (band). However, the discontinuity orientation cannot be determined uniquely unless some additional assumptions, e.g., Mohr's maximization postulate considered in this work, are made. With respect to the strategies dealing with the traction continuity condition between the bulk stresses and tractions across the discontinuity (band), two approaches can be identified. If the traction continuity condition is enforced in weak (average) form, the strong/regularized discontinuity approaches follow. In this approach, the displacement jumps (or equivalently, the inelastic deformations) are retained as independent variables, and constitutive models for both the bulk and discontinuity (band) should be fed. Alternatively, being enforced in strong (point-wise) form, the traction continuity condition coincides with the classical static constraint. This property allows eliminating the kinematic unknowns (i.e., displacement jumps or inelastic deformations) associated with the discontinuity (band) from the governing equations. In the resulting embedded/smeared discontinuity approaches, an overall inelastic constitutive model fulfilling the static constraint suffices.

In order to check whether above strain localization can occur and identify its consequences on the resulting approaches, the second methodology is considered. The kinematic constraint guaranteeing stress boundedness/continuity upon strain localization is established for general inelastic softening solids. It is then applied to a unified elastoplastic damage model with the inelastic evolution laws characterized by a dissipative flow tensor. For the discontinuity kinematics to be reproduced in an appropriate manner, the tensorial components of the dissipative flow tensor in the directions orthogonal to the discontinuity orientation have to vanish upon strain localization. Satisfaction of this kinematic constraint allows introducing a traction-based failure criterion and developing a localized cohesive model for the discontinuity (band), justifying the first methodology. Dependent on the strategies dealing with the discontinuity orientation and failure criterion, two dual (formally identical) but not necessarily equivalent approaches are identified. 
For a given traction-based failure criterion and a known discontinuity orientation, the resulting elastoplastic damage model is equivalent to the embedded/smeared discontinuity models discussed before. Alternatively, for a given stress-based failure criterion, the discontinuity orientation and associated traction-based counterpart are consistently determined from the kinematic constraint, resulting in the projected discontinuity model.

The bi-directional connections between the aforementioned traction- and stress-based approaches are explored in general 3D cases. Particularly, the equivalence between Mohr's maximization postulate for a traction-based failure criterion and the kinematic constraint for a stress-based one is established. This equivalence makes it possible to bridge the gap between traction- and stress-based approaches. In the plane stress condition, the discontinuity orientation and the corresponding stress-/traction-based failure criteria are explicitly given in closed form. A generic stress-based failure criterion is then analyzed in a unified manner. Depending on the involved parameters, the projected tractionbased failure criterion can be either an ellipse, a parabola, a hyperbola or the product of two straight lines, recovering most of those adopted in the literature, e.g., the classical Von Mises $\left(J_{2}\right)$, Drucker-Prager and Mohr-Coulomb criteria, as particular cases. In particular, a traction-based failure criterion of hyperbolic type, widely adopted for the modeling of mixed mode localized failure in quasi-brittler materials, is obtained naturally. More importantly, owing to the established equivalence, the involved model parameters can be identified from available standard experimental data, so that the deficiency hindering its application in practice is largely facilitated.

\section{Acknowledgments}

Support from the National Natural Science Foundation of China (51222811, 51008130) and the State Key Laboratory of Subtropic Building Science (2015ZB24) to the first author (J.Y. Wu) is acknowledged. Support from the Spanish Ministry of Economy and Competitivity under the project Enhanced Accuracy Computational Framework for Strain Localization and Failure Mechanisms (EACY) - is acknowledged by the second author (M. Cervera).

\section{References}

[1] Armero, F., 1999. Large-scale modeling of localized dissipative mechanisms in a local continuum: applications to the numerical simulation of strain localization in rate-dependent inelastic solids. Mech. Cohes.-Frict. Mater., 4: 101-131.

[2] Armero, F. and Oller, S., 2000. A general framework for continuum damage models. I: Infinitesimal plastic damage models in stress space. Int. J. Solids Structures, 37: 7409-7464.

[3] Barenblatt, G.I., 1959. The formation of equilibrium cracks during brittle fracture. General ideas and hypotheses. Axiallysymmetric cracks. J. Applied Mathematics and Mechanics, 23: 622-636.

[4] Barenblatt, G., 1962. The mathematical theory of equilibrium cracks in brittle fracture. Adv. Appl. Mech., 7: 55-129.

[5] Bažant, Z.P. and Oh, B.H., 1983. Crack band theory for fracture of concrete. Mater. Struct. (RILEM, Paris), 16: 155-177.

[6] Benvenuti, E., Tralli, A., 2012. Simulation of finite-width process zone in concrete-like materials by means of a regularized extended finite element model. Comput. Mech., 50: 479-497. 
[7] Borré G. and Maier, G., 1989. On linear versus nonlinear flow rules in strain localization analysis. Meccanica, $24:$ 36-41.

[8] Camacho G.T. and Ortiz M., 1996. Computational modeling of impact damage in brittle materials. Int. J. Solids Structures, 33: $2899-2938$

[9] Carol, I., Prat, P., and López, C., 1997. Normal/shear cracking model: Application to discrete crack analysis. J. Eng. Mech., ASCE, 123(8): 765-773.

[10] Carol, I., Rizzi, E., Willam, K., 1994. A unified theory of elastic degradation and damage based on a loading surface. Int. J. Solids Structures, 31(20): 2835-2865.

[11] Cazes, F., Coret, M., Combescure, A. and Gravouil, A., 2009. A thermodynamic method for the construction of a cohesive law from a nonlocal damage model. Int. J. Solids Structures, 46: 1476-1490.

[12] Cazes, F., Simatos, A., Coret, M. and Combescure, A., 2010. A cohesive zone model which is energetically equivalent to a gradient-enhanced coupled damage-plasticity model. European Journal of Mechanics A/Solids, 29: 976-989.

[13] Cervera, M., 2008a. A smeared-embedded mesh-corrected damage model for tensile cracking. Int. J. Numer. Meth. Engng., 76: $1930-1954$.

[14] Cervera, M., 2008b. An othortropic mesh corrected model. Comput. Methods Appl. Mech. Engrg., 197: 1603-1619.

[15] Cervera, M., Chiumenti, M. and Di Capua, D., 2012. Benchmarking on bifurcation and localization in $J_{2}$ plasticity for plane stress and plane strain conditions. Comput. Methods Appl. Mech. Engrg., 241-244: 206-224.

[16] Cervera, M. and Chiumenti, 2006a. Smeared crack approach: back to the original track. Int. J. Numer. Anal. Meth. Geomech., 30: 1173-1199.

[17] Cervera, M. and Chiumenti, 2006b. Mesh objective tensile cracking via a local continuum damage model and a crack tracking technique. Comput. Methods Appl. Mech. Engrg., 196: 304-320.

[18] Cervera, M., Chiumenti, M. Benedetti, L. and Codina, R., 2015. Mixed stabilized finite element methods in nonlinear solid mechanics. Part III: Compressible and incompressible plasticity. Comput. Methods Appl. Mech. Engrg., 285: 752-775.

[19] Cervenka, J., 1994. Discrete Crack Modelling in Concrete Structures. Ph.D. Thesis, University of Colorado, Boulder.

[20] Chen, W.F., 1994. Constitutive Equations for Engineering Materials: Plasticity and Modeling, vol. 2. Elsevier, Amsterdam.

[21] Comi, C. and Perego, U., 2001. Fracture energy based bi-dissipative damage model for concrete. Int. J. Solids Structures, 38 : 6427-6454.

[22] Dugdale, D., 1960. Yielding of steel sheets containing slits. J. Mech. Phys. Solids, 8: 100-108.

[23] Golub, G.H. and van Loan, C.F., 1996. Matrix computations, third edition (page 50). The John Hopkins University Press, Baltimore.

[24] Govindjee, S.D, Kay, G.J. and Simò, J.C., 1995. Anisotropic modeling and numerical simulation of brittle damage in concrete.. Int. J. Numer. Meth. Engng, 38: 3611-3633.

[25] Hill, R., 1958. General theory of uniqueness and stability of elasto-plastic solids. J. Mech. Phys. Solids, 6: 236-249.

[26] Hill, R., 1962. Acceleration waves in solids. J. Mech. Phys. Solids, 10: 1-16. 
[27] Hillerborg, A., Modeer, M. and Petersson, P.E., 1976. Analysis of crack formation and crack growth in concrete by means of fracture mechanics and finite elements. Cement Concrete Res., 6: 773-782.

[28] Itskov, M., 2007. Tensor algebra and tensor analysis for engineers: With applications to continuum mechanics. Springer, Berlin.

[29] Jirásek, M. and Zimmermann, T., 2001. Embedded crack model. I: Basic formulation. Internat. J. Numer. Methods Engrg., 50(6): 1269-1290.

[30] Krajcinovic, D., 2003. Damage Mechanics. Elsevier B.V., Netherlands.

[31] Kupfer, H., Hilsdorf, H.K. and Rüsch, H., 1969. Behavior of concrete under biaxial stresses. ACI J., 66(8): 656-666.

[32] Lee, S.K., Song, Y.C. and Han, S.H., 2003. Biaxial behavior of plain concrete of nuclear containment building. Nucl. Eng. Des. 227, 143153.

[33] Li, W.Z., Guo, Z.H., 1991. Experimental research for strength and deformation of concrete under biaxial tension-compression loading (in Chinese). Journal of Hydraulic Engineering, 8: 51-56.

[34] Malvern, L.E., 1969. Introduction to the mechanics of a continuous medium. Prentice-Hall International, New Jersey.

[35] Mazars, J. and Pijaudier-Cabot, G., 1996. From damage to fracture mechanics and conversely: a combined approach. Int. J. Solids Structures, 33(20-22): 3327-3342.

[36] Meschke, G, Lackner, R. and Mang, H.A., 1998. An anisotropic elastoplastic damage model for plain concrete. Int. J. Numer. Meth. Engng., 42: 703-727.

[37] Moës, N., Dolbow, J. and Belytschko, T., 1999. A finite element method for crack growth without remeshing. Int. J. Numer. Meth. Engng, 46: 131-150.

[38] Mohr, O., 1900. Welche Umstände bedingen die Elastizitätsgrenze und den Bruch eines Materials? Civilingenieur: Zeitschrift des Vereins deutscher Ingenieure, VDI 44/45: 1524-1530; 1572-1577.

[39] Mosler, J., 2005. On advanced solution strategies to overcome locking effects in strong discontinuity approaches. Int. J. Numer. Meth. Engng., 63: 1313-1341.

[40] Most, T. and Bucher, C., 2007. Energy-based simulation of concrete cracking using an improved mixed-mode cohesive crack model within a meshless discretization. Int. J. Numer. Anal. Meth. Geomech., 31: 285-305

[41] Ngo, D., Scordelis, A.C., 1967. Finite element analysis of reinforced concrete beams, ACI J., 64(14): 152-163.

[42] Ohlsson, U. and Olofsson, T., 1997. Mixed-mode fracture and anchor bolts in concrete analysis with inner softening bands. J. Eng. Mech., ASCE, 123: 1027-1033.

[43] Oliver, J., 1989. A consistent characteristic length for smeared cracking models. Int. J. Numer. Meth. Engng, 28: 461-474.

[44] Oliver, J., 1996. Modeling strong discontinuities in solid mechanics via strain softening contitutive equations. Part I: Fundaments \& Part II: Numerical simulation. Int. J. Numer. Meth. Engng, 39: 3575-3623.

[45] Oliver, J., Cervera, M. and Manzoli, O. 1998. On the use of strain-softening models for the simulation of strong discontinuities in solids. Material instabilities in solids: 107-123, Wiley.

[46] Oliver, J. Cervera, M. and Manzoli, O., 1999. Strong discontinuities and continuum plasticity models: the strong discontinuity 
approach. Int. J. Plast., 15: 319-351.

[47] Oliver, J., 2000. On the discrete constitutive models induced by strong discontinuity kinematics and continuum constitutive equations. Int. J. Solids Structures, 37: 7207-7229.

[48] Oliver, J., Huespe, A.E., Pulido, M.D.G., Chaves, E., 2002. From continuum mechanics to fracture mechanics: the strong discontinuity approach. Eng. Fract. Mech., 69: 113-136.

[49] Oliver, J., Huespe, A.E., Pulido, M.D.G., Blanco, S. and Linero, D., 2006. On the fracture models determined by the continuum-strong discontinuity approach. Int. J. Fract., 137: 211-229.

[50] Oliver, J., Huespe, A.E. and Dias, I.F., 2012. Strain localization, strong discontinuities and material fracture: Matches and mismatches. Comput. Methods Appl. Mech. Engrg., 241-244: 323-336.

[51] Oliver, J., Huespe, A.E. and Sànchez, P.J., 2006. A comparative study on finite elements for capturing strong discontinuities: E-FEM vs X-FEM. Comput. Methods Appl. Mech. Engrg., 195: 4732-4752.

[52] Ohtani, Y. and Chen, W.F., 1988. Multple hardening plasticity for concrete material. J. Eng. Mech., ASCE, 114: 1890-1910.

[53] Ortiz., M., 1985. A constitutive theory for the inelastic behavior of concrete. Mech. Mater., 4: 76-93.

[54] Ottosen, N. and Runesson, K., 1991. Discontinuous bifurcations in a non-associated Mohr material. Mech. Mater., 12: 255265.

[55] Patzák, B. and Jirásek, M., 2003. Process zone resolution by extended finite elements. Engineering Fracture Mechanics, 70: 957-977.

[56] Pietruszczak, S., and Mroź, Z., 1981. Finite-element analysis of deformation of strain-softening materials. Int. J. Numer. Methods Eng., 17: 327-334.

[57] Pandolfi, A., Krysl, P. and Ortiz, M., 1999. Finite element simulation of ring expansion and fragmentation: the capturing of length and time scales through cohesive models of fracture. Int. J. Fracture, 95: 279-297.

[58] Planas, J., Elices, M. and Guinea, G., 1993. Cohesive cracks versus nonlocal models: closing the gap. Int. J. Fracture, 63(2): 173-187.

[59] Rashid, Y., 1968. Analysis of prestressed concrete pressure vessels. Nucl. Eng. Des., 7: 334-344.

[60] Rice, J.R. and Rudnicki, J.W., 1980. A note on some features of the theory of localization of deformation. Int. J. Solids Structures, 16: 597-605.

[61] Roscoe, K.H., 1970. The influence of strains in soil mechanics, 10th Rankine Lecture. Géotechnique, 20: 129-180.

[62] Rots, J.G., et al., 1985. Smeared crack approach and fracture localization in concrete. Heron, 30: 1-47.

[63] Rots, J.G., 1988. Computational modeling of concrete fracture. Doctoral Dissertation, Delft University of Technology, Delft, The Netherlands.

[64] Rots, J.G., Blaauwendraad, J., 1989. Crack models for concrete: discrete or smeared? fixed, multi-directional or rotating? Heron, 34(1): 1-59.

[65] Rudnicki, J.W. and Rice, J.R., 1975. Conditions of the localization of deformation in pressure-sensitive dilatant material. J. Mech. Phys. Solids, 23: 371-394. 
[66] Runesson, K., Ottosen, N.S. and Peric, D., 1991. Discontinuous bifurcations of elastic-plastic solutions at plane stress and plane strain. Int. J. Plast., 7: 99-121.

[67] Simó, J.C. and Ju, J.W., 1987. Strain- and stress-based continuum damage models. I: Formulation; II: Computational aspects. Int. J. Solids Structure, 23(7): 821-869.

[68] Simó, J.C. and Oliver, J., 1994. A new approach to the analysis and simulation of strain softening in solids. In: Z.P. Bažant et al. (eds.), Fracture and Damage in Quasibrittle Structures, E \& FN Spon: 25-39.

[69] Simó, J.C., Oliver, J. and Armero, F., 1993. An analysis of strong discontinuities induced by strain-softening in rateindependent inelastic solids. Comput. Mech. , 12: 277-296.

[70] Stankowski, T., Runesson, K., Sture, S., 1993. Fracture and slip of interfaces in cementitious composites. I: Characteristics, II: Implementation. J. Engrg. Mech., ASCE, 90: 845-867.

[71] Steinmann, P., Willam, K., 1991. Localization within the framework of micropolar elastoplasticity. Advances in Continuum Mechanics, VI: 296-313

[72] Thomas, T.Y., 1961. Plastic Flow and Fracture of Solids. Academic Press, New York.

[73] Weihe, S., Kröplin, B. and de Borst R., 1997. Classification of smeared crack models based on material and structural properties. Int. J. Solids Structures, 35(5): 467-484.

[74] Wells, G.N. and Sluys, L.J., 2001a. On the conceptual equivalence of embedded strong discontinuity and smeared crack formulations. Heron, 46(3): 181-189.

[75] Wells, G.N. and Sluys, L.J., 2001b. A new method for modelling cohesive cracks using finite elements. Int. J. Numer. Meth. Engng., 50: 2667-2682.

[76] Wu, J. Y., 2011. Unified analysis of enriched finite elements for modeling cohesive cracks. Comput. Methods Appl. Mech. Engrg., 200(45-46): 3031-3050.

[77] Wu, J. Y., Cervera, M., 2013. Strain localization in elastoplastic damage solids. In: Proceeding of International Symposium on Innovation \& Sustainability of Structures in Civil Engineering (ISISS-2013), Harbin, China.

[78] Wu, J. Y., Cervera, M., 2014. On the stress continuity condition for strain localization in softening solids. In: Proceeding of 11th World Congress On Computational Mechanics (WCCM-2014), Barcelona, Spain.

[79] Wu, J. Y., Cervera, M., 2014b. Strain localization and failure mechanics for elastoplastic damage solids. Monograph CIMNE, M147, Barcelona, Spain.

[80] Wu, J.Y. and Xu, S.L., 2011. An augmented multicrack elastoplastic damage model for tensile cracking. Int. J. Solids Structures, 48: 2511-2528.

[81] Wu, J. Y., Li, F. B. and Xu, S. L., 2015. Extended embedded finite elements with continuous displacement jumps for the modeling of localized failure in solids. Comput. Methods Appl. Mech. Engrg., 285: 346-378. 


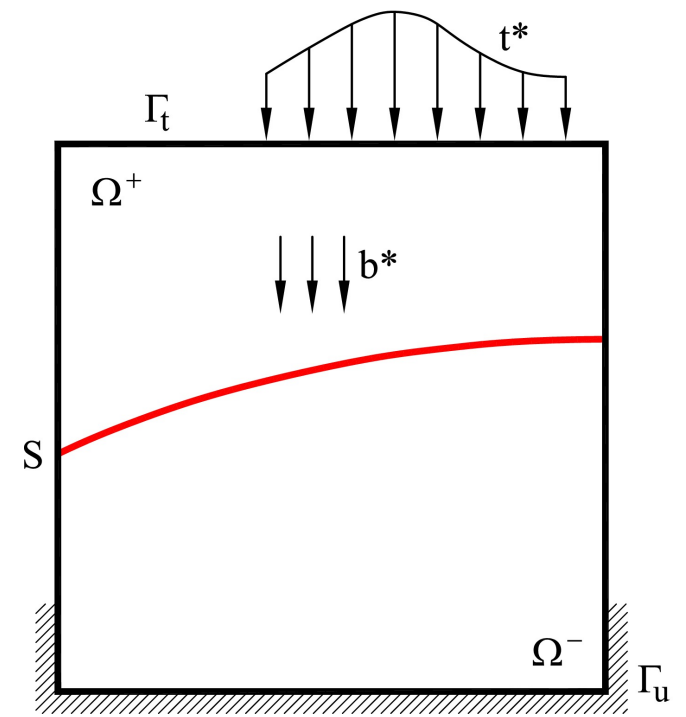

Figure 1: Problem setting in an elastic solid medium with an internal discontinuity

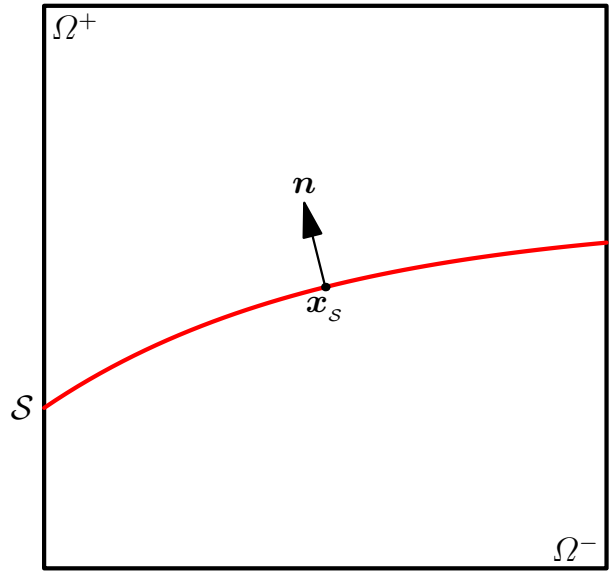

(a) Strong discontinuity

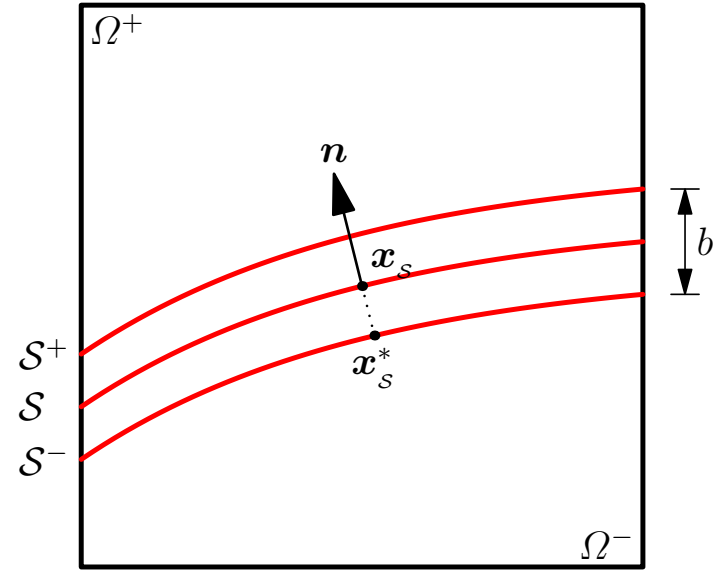

(b) Regularized discontinuity

Figure 2: Strong and regularized discontinuities in a solid 


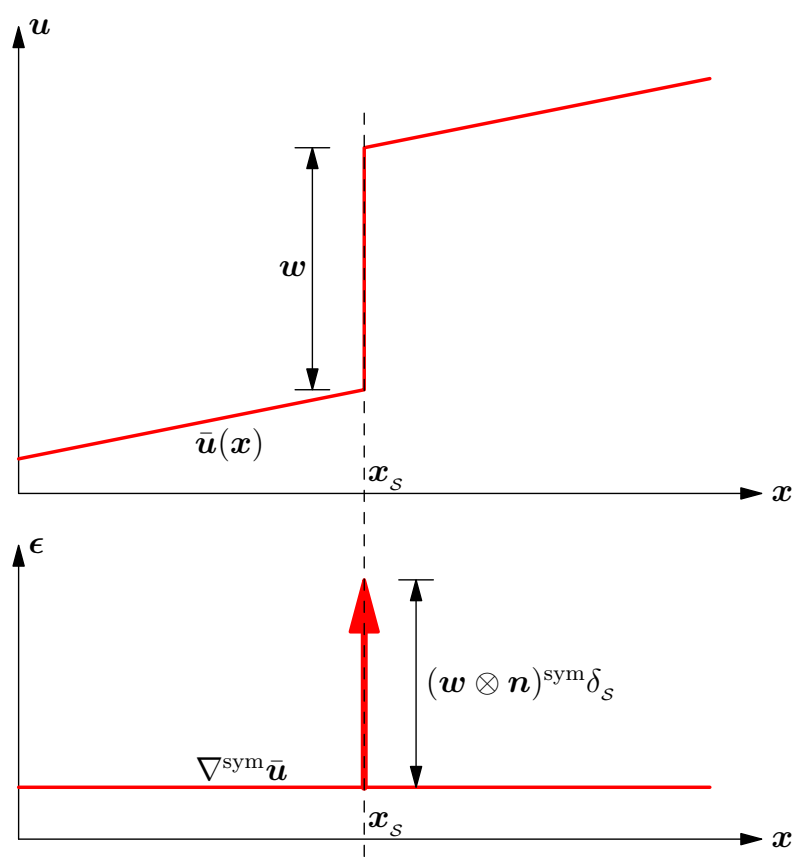

(a) Displacement/strain fields around a strong discontinuity

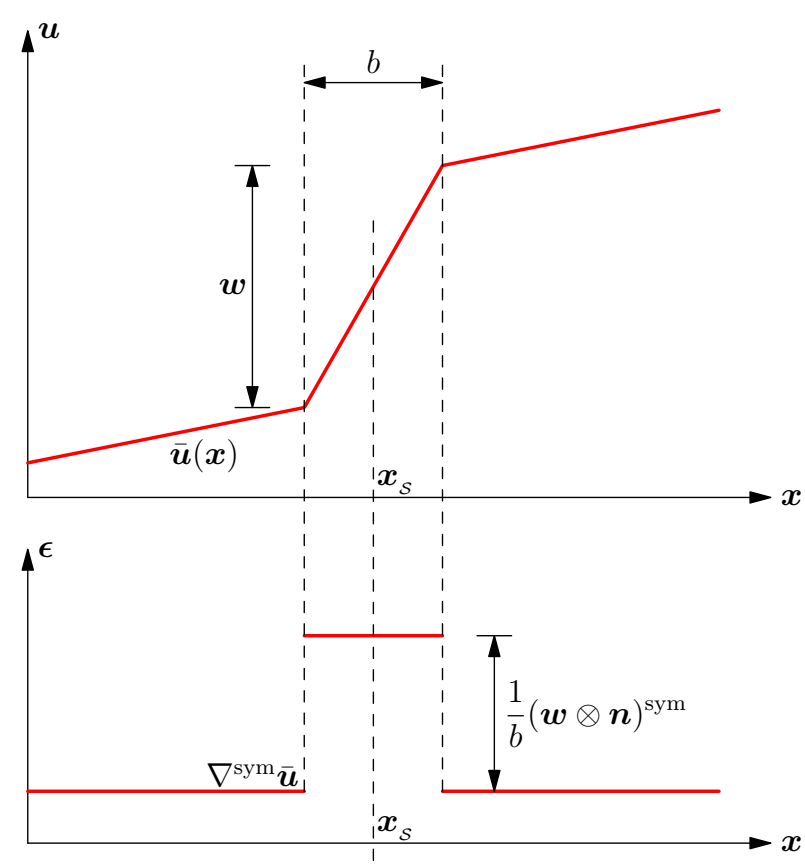

(b) Displacement/strain fields around a regularized discontinuity

Figure 3: Kinematics of strong/regularized discontinuities

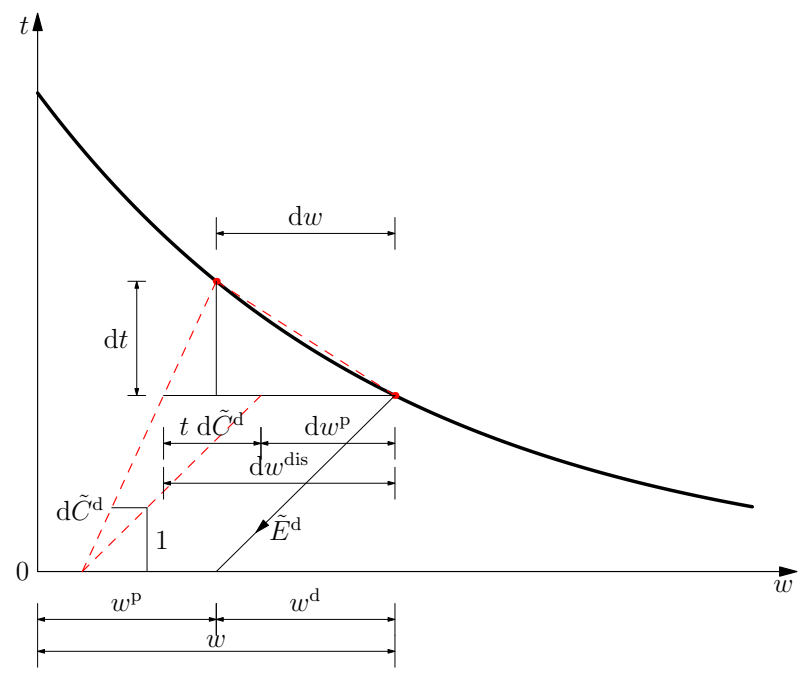

(a) Dissipative jump rate and its damage/plastic components

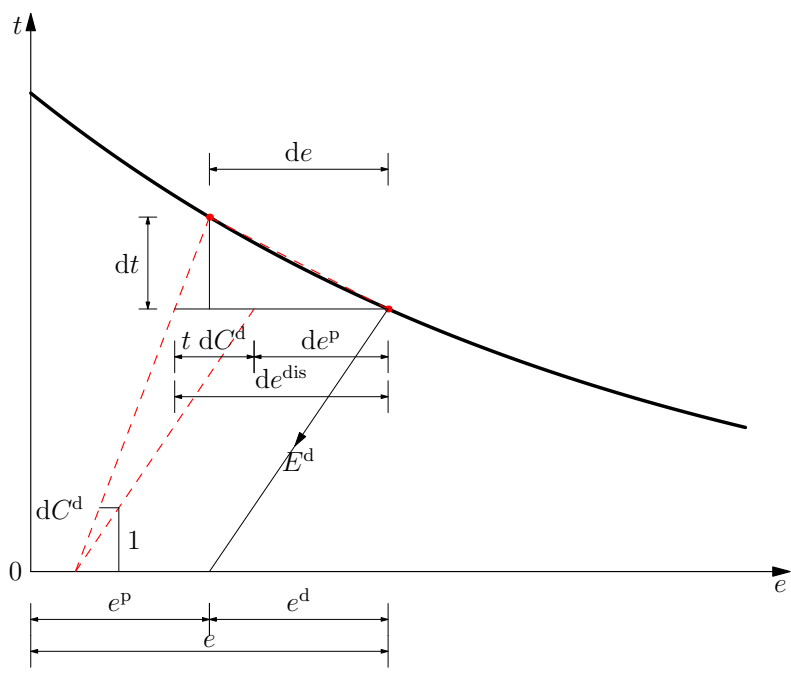

(b) Dissipative deformation rate and its damage/plastic components

Figure 4: 1D definition of the dissipative jump rate (or deformation rate) for an increment deformation 


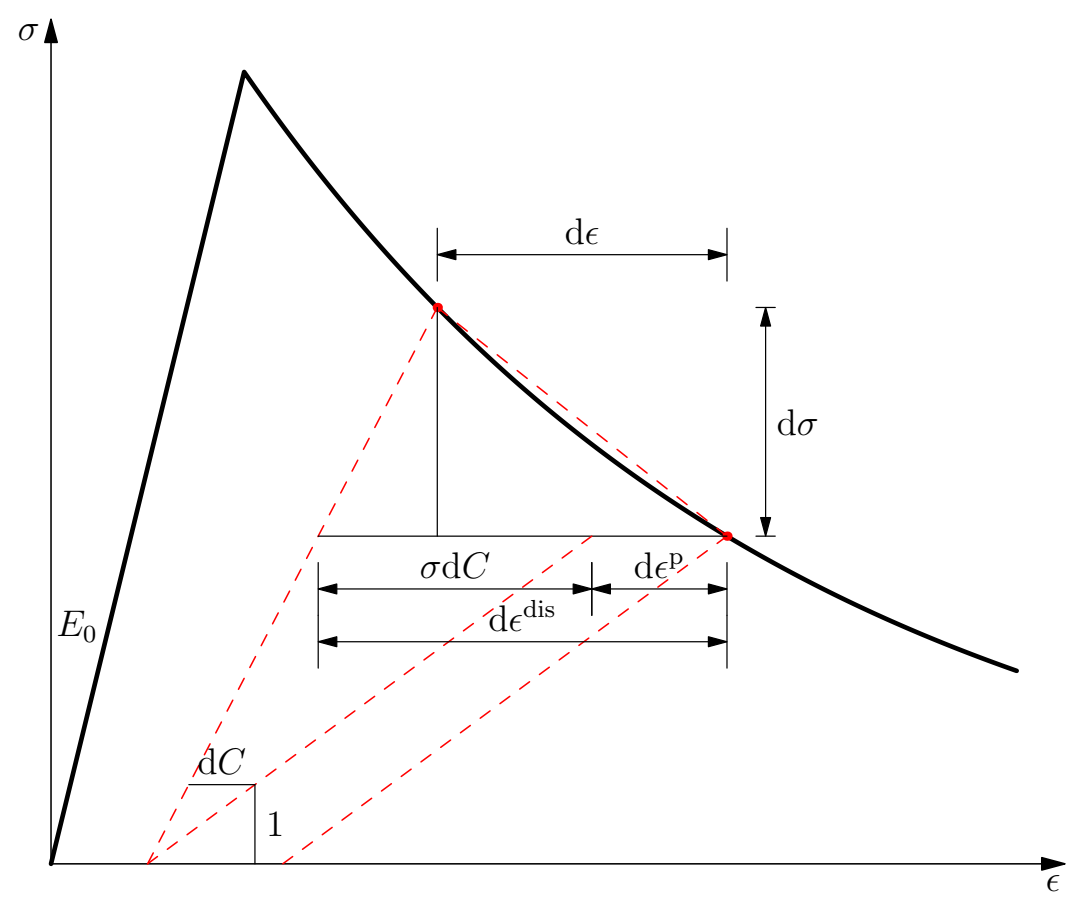

Figure 5: 1D definition of the dissipative strain rate and its damage/plastic components for an infinitesimal increment strain

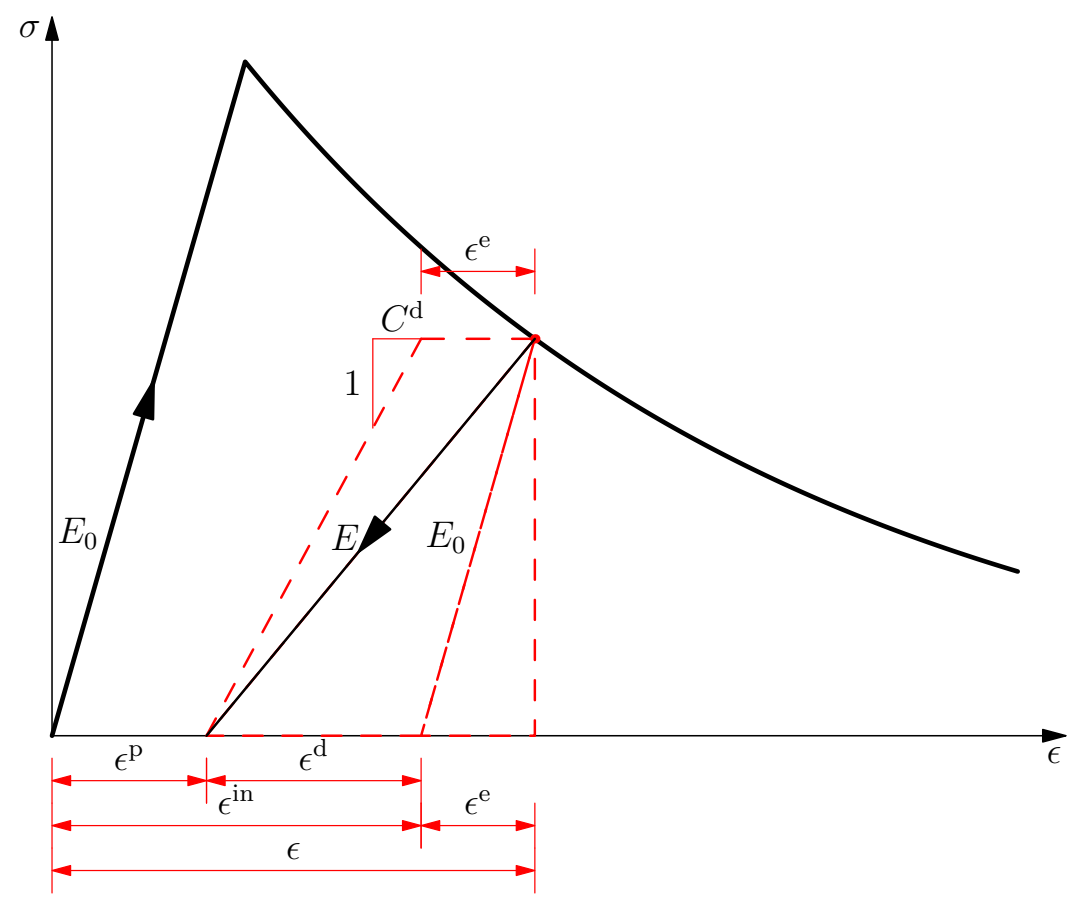

Figure 6: 1D stress vs. strain relation of the elastoplastic damage model: different kinematic decompositions 


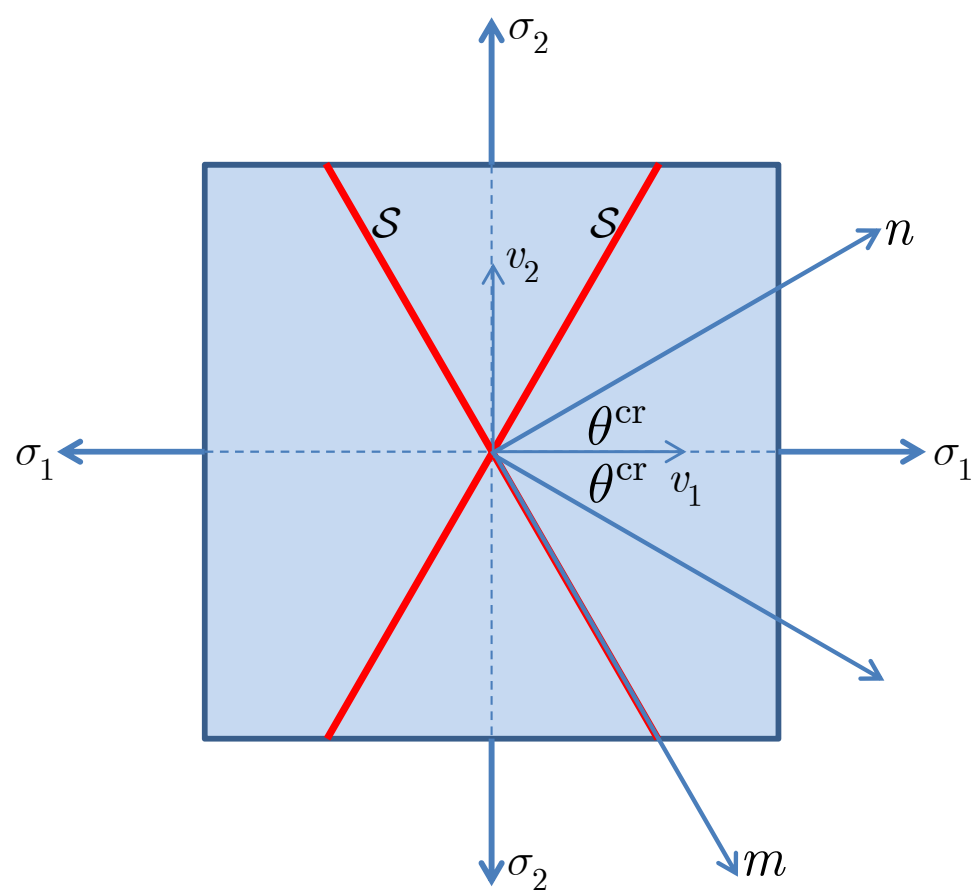

Figure 7: Definition of the discontinuity angle in $2 \mathrm{D}$ cases

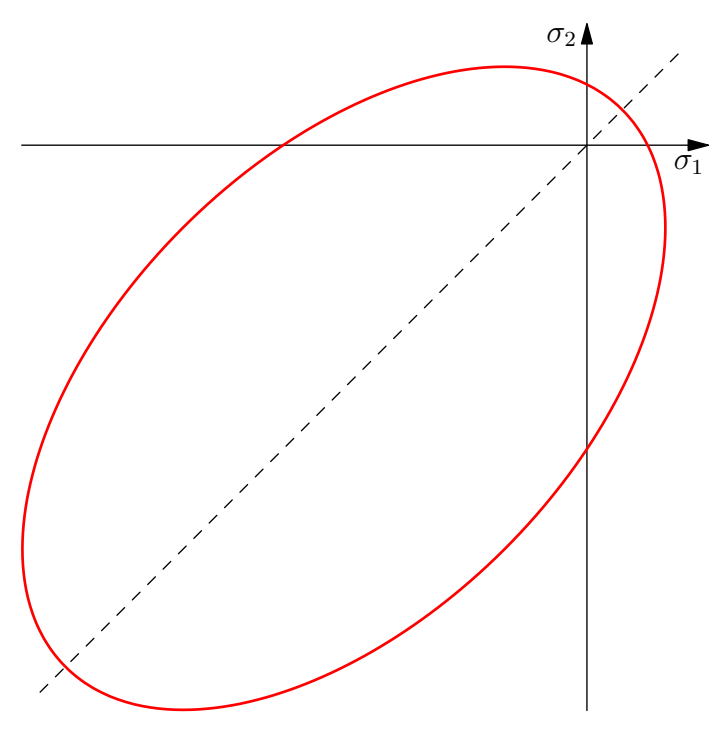

(a) Stress-based failure surface

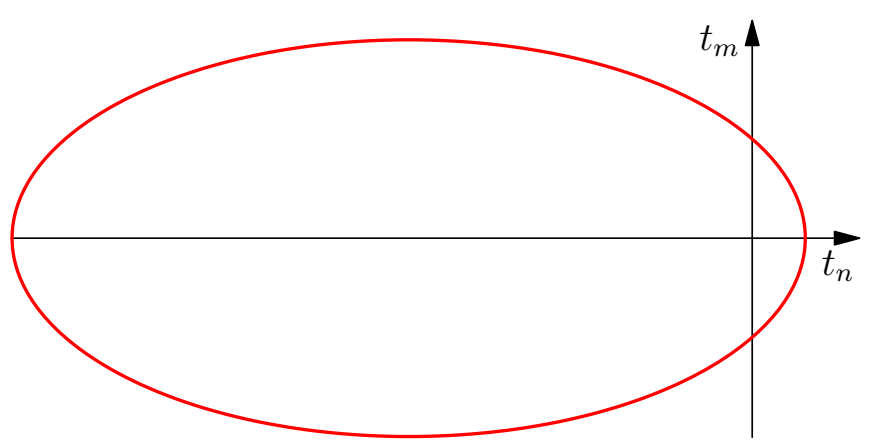

(b) Traction-based failure surface

Figure 8: Elliptic stress- and traction-based failure surfaces in plane stress $\left(\rho=5.0, A_{0}=0.0\right)$ 


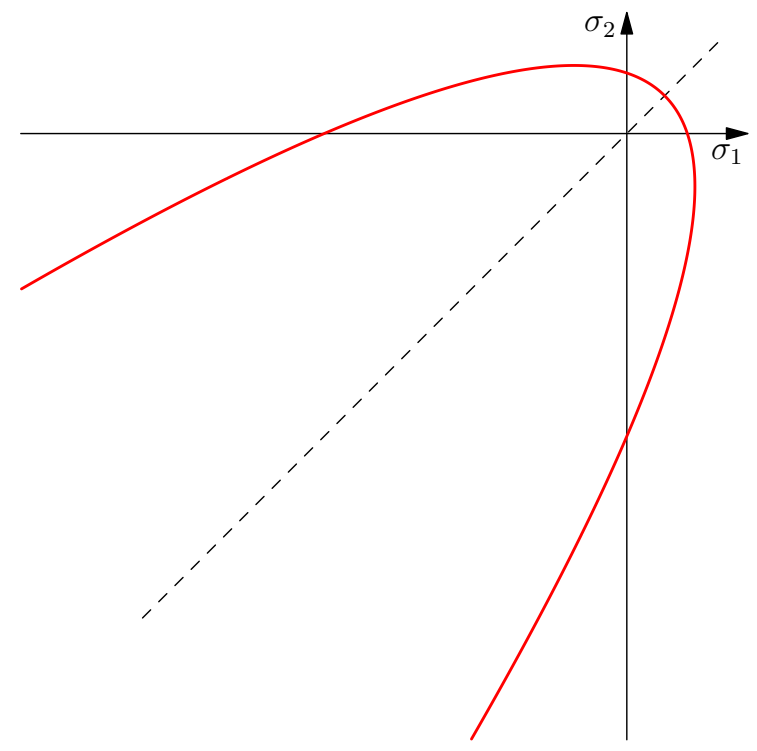

(a) Stress-based failure surface

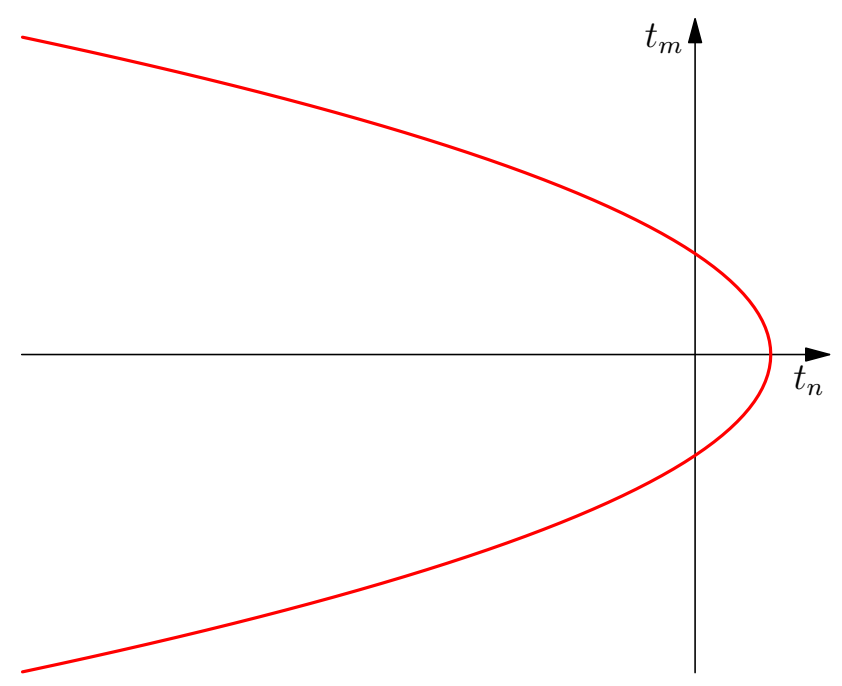

(b) Traction-based failure surface

Figure 9: Parabolic stress- and traction-based failure surfaces in plane stress $\left(\rho=5.0, A_{0}=0.5\right)$

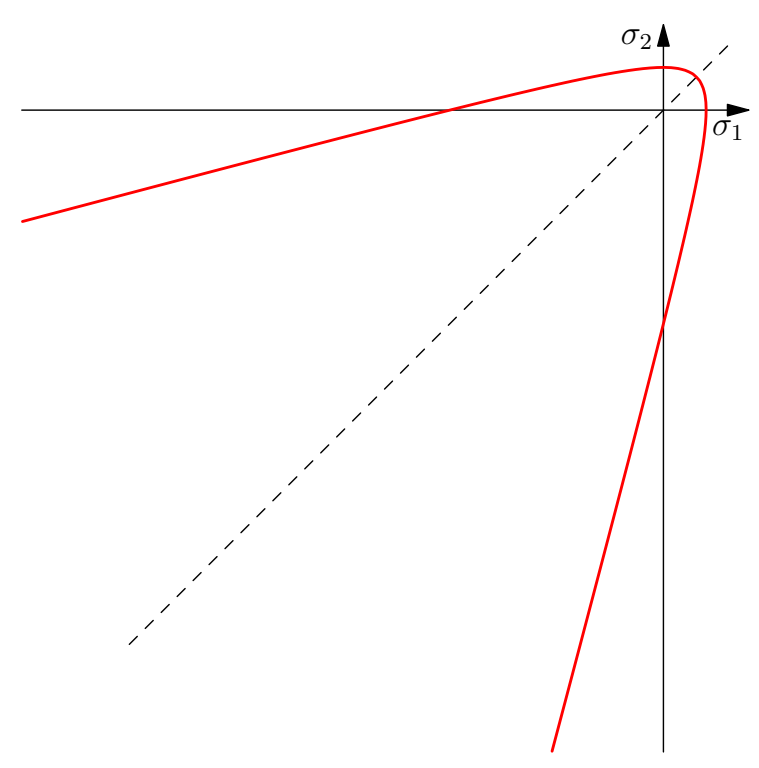

(a) Stress-based failure surface

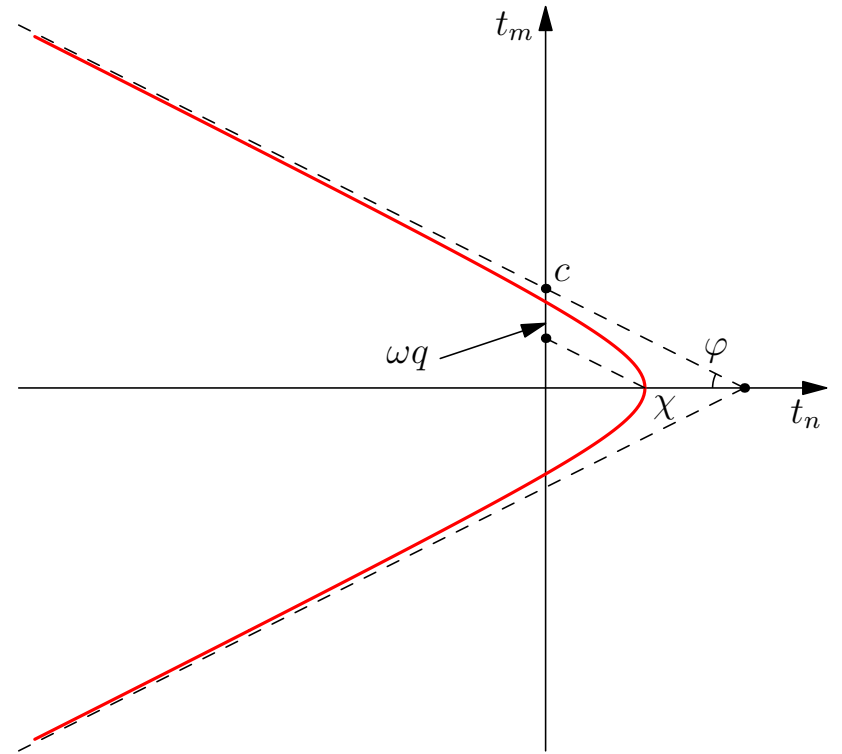

(b) Traction-based failure surface

Figure 10: Hyperbolic stress- and traction-based failure surfaces in plane stress $\left(\rho=5.0, A_{0}=1.0\right)$ 


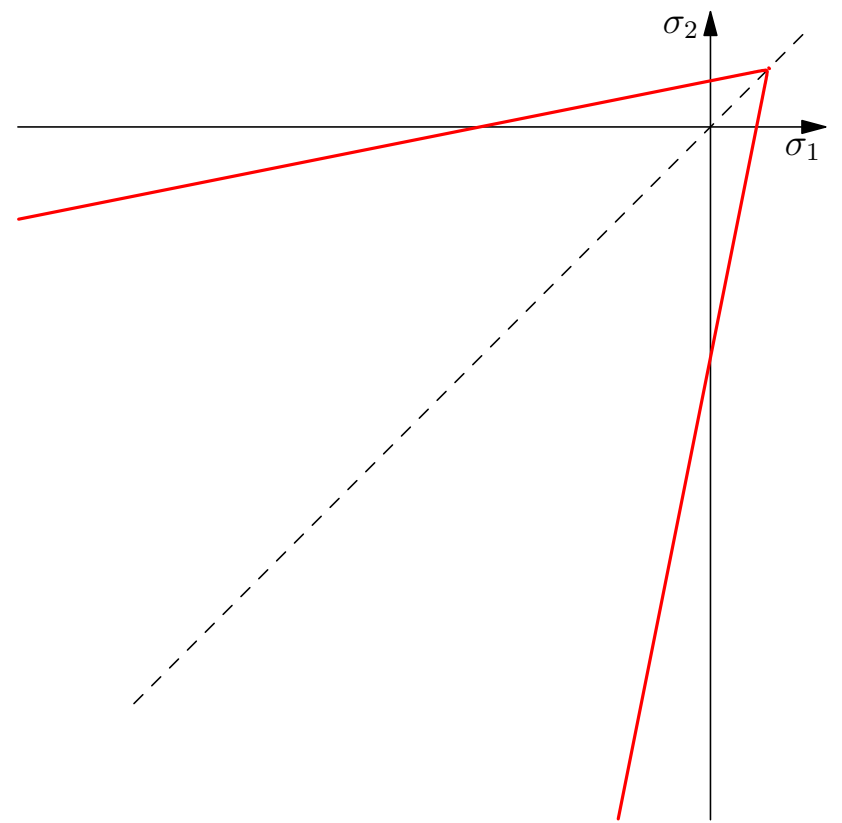

(a) Stress-based failure surface

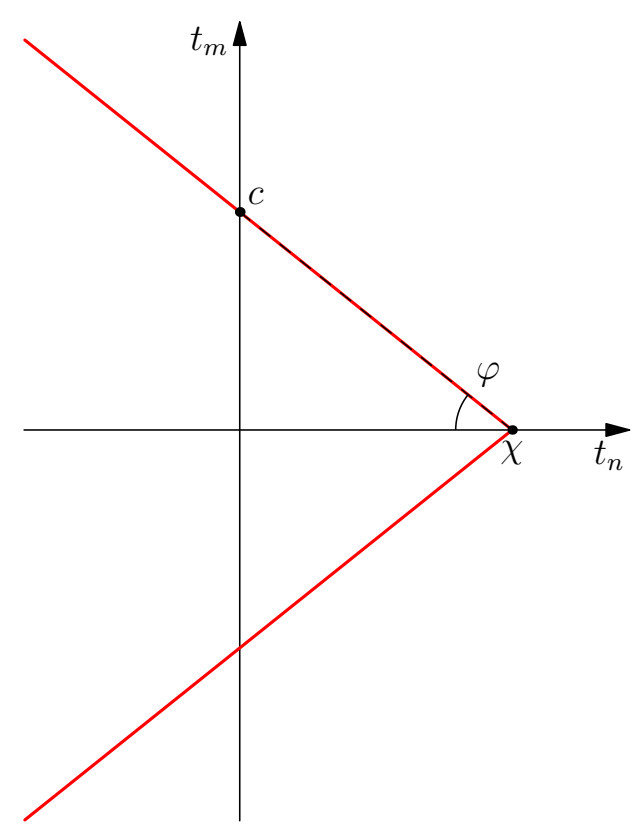

(b) Traction-based failure surface

Figure 11: Bilinear stress- and traction-based failure surfaces in plane stress $\left(\rho=5.0, A_{0}=7 / 6\right)$

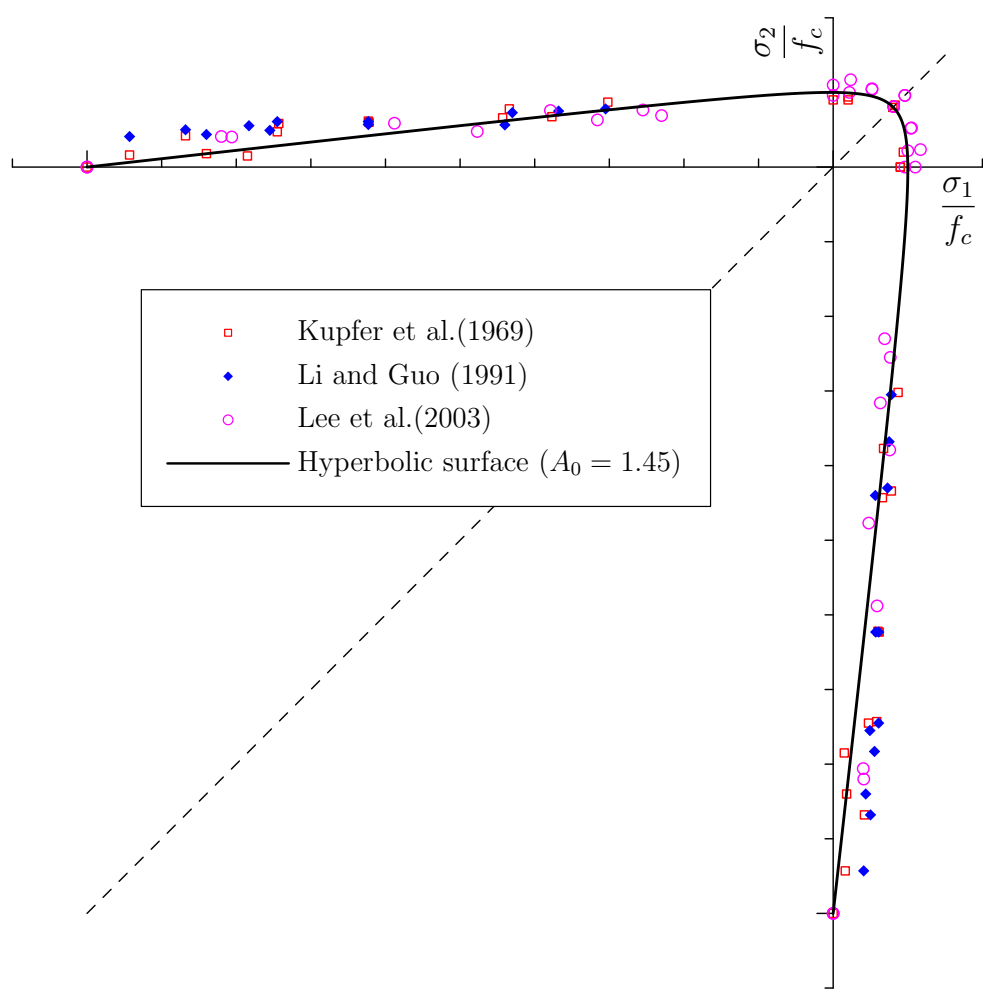

(a) Biaxial strength envelope

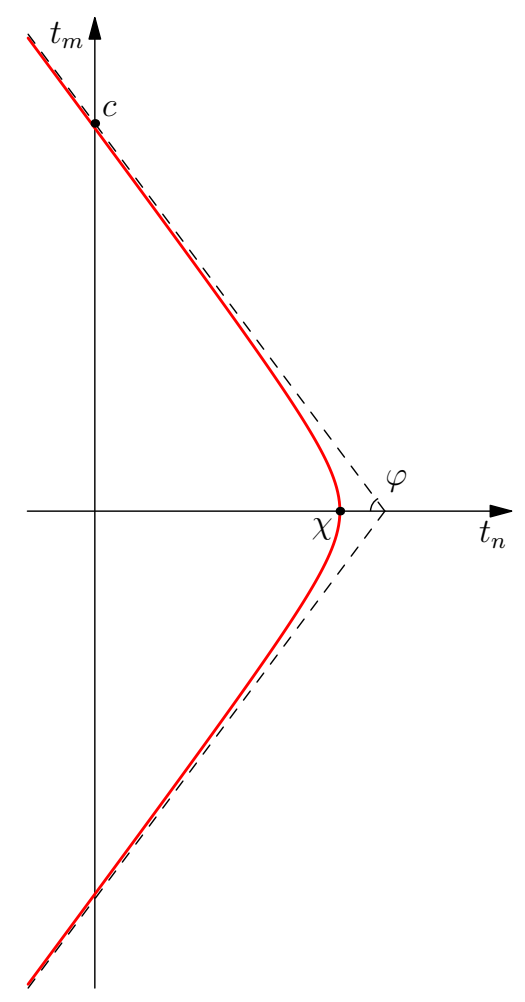

(b) Hyperbolic traction-based failure surface

Figure 12: Stress- and traction-based failure surface typical for concrete in plane stress 